\title{
Transcriptome profiling of non-climacteric 'yellow' melon during ripening: insights on sugar metabolism
}

Michelle Orane Schemberger ${ }^{1}$, Marília Aparecida Stroka ${ }^{1}$, Letícia Reis ${ }^{1}, K^{\prime}$ Kamila Karoline de Souza Los ${ }^{1}$, Gillize Aparecida Telles de Araujo ${ }^{1}$, Michelle Zibetti Tadra Sfeir ${ }^{3}$, Carolina Weigert Galvão², Rafael Mazer Etto², Amanda Regina Godoy Baptistão ${ }^{1}$ and Ricardo Antonio Ayub ${ }^{1 *}$ (D)

\begin{abstract}
Background: The non-climacteric 'Yellow' melon (Cucumis melo, inodorus group) is an economically important crop and its quality is mainly determined by the sugar content. Thus, knowledge of sugar metabolism and its related pathways can contribute to the development of new field management and post-harvest practices, making it possible to deliver better quality fruits to consumers.

Results: The RNA-seq associated with RT-qPCR analyses of four maturation stages were performed to identify important enzymes and pathways that are involved in the ripening profile of non-climacteric 'Yellow' melon fruit focusing on sugar metabolism. We identified 895 genes 10 days after pollination (DAP)-biased and 909 genes 40 DAP-biased. The KEGG pathway enrichment analysis of these differentially expressed (DE) genes revealed that 'hormone signal transduction', 'carbon metabolism', 'sucrose metabolism', 'protein processing in endoplasmic reticulum' and 'spliceosome' were the most differentially regulated processes occurring during melon development. In the sucrose metabolism, five DE genes are up-regulated and 12 are down-regulated during fruit ripening.
\end{abstract}

Conclusions: The results demonstrated important enzymes in the sugar pathway that are responsible for the sucrose content and maturation profile in non-climacteric 'Yellow' melon. New DE genes were first detected for melon in this study such as invertase inhibitor LIKE $3(\mathrm{Cm} / \mathrm{NH} 3)$, trehalose phosphate phosphatase (CMTPP1) and trehalose phosphate synthases (CMTPS5, CMTPS7, CMTPS9). Furthermore, the results of the protein-protein network interaction demonstrated general characteristics of the transcriptome of young and full-ripe melon and provide new perspectives for the understanding of ripening.

Keywords: Cucumis melo, RNA-seq, Sucrose, Fruit ripening, Gene expression

\footnotetext{
* Correspondence: rayub@uepg.br

'Laboratório de Biotecnologia Aplicada a Fruticultura, Departamento de Fitotecnia e Fitossanidade, Universidade Estadual de Ponta Grossa, Av. Carlos Cavalcanti, 4748, Ponta Grossa, Paraná 84030-900, Brazil

Full list of author information is available at the end of the article
}

(c) The Author(s). 2020 Open Access This article is licensed under a Creative Commons Attribution 4.0 International License, which permits use, sharing, adaptation, distribution and reproduction in any medium or format, as long as you give appropriate credit to the original author(s) and the source, provide a link to the Creative Commons licence, and indicate if changes were made. The images or other third party material in this article are included in the article's Creative Commons licence, unless indicated otherwise in a credit line to the material. If material is not included in the article's Creative Commons licence and your intended use is not permitted by statutory regulation or exceeds the permitted use, you will need to obtain permission directly from the copyright holder. To view a copy of this licence, visit http://creativecommons.org/licenses/by/4.0/ The Creative Commons Public Domain Dedication waiver (http://creativecommons.org/publicdomain/zero/1.0/) applies to the data made available in this article, unless otherwise stated in a credit line to the data. 


\section{Background}

Melon (Cucumis melo L., Cucurbitaceae) is an economically important fruit crop worldwide that has an extensive polymorphism being classified into 19 botanical groups $[1,2]$. This high intra-specific genetic variation is reflected in fruit ripening differences. In this regard, melon fruits present both climacteric and nonclimacteric phenotypes. Climacteric fruits are characterized by a respiration peak followed by the autocatalytic synthesis of ethylene, strong aroma, orange pulp, ripening abscission and short shelf life with rapid loss of firmness and taste deterioration (e.g. cantalupensis and reticulatus melon groups). On the other hand, nonclimacteric melon (e.g. inodorus melon group) has little ethylene synthesis, white pulp, low aroma, no ripening abscission and a longer shelf life [3-7].

During the ripening process, fruits undergo several biochemical and physiological changes that are reflected in their organoleptic profile, of which the alteration in sucrose accumulation is a determining characteristic in melon quality and consumption $[6,8,9]$. This characteristic is a developmentally regulated process that is related to gene regulation, hormonal signalling and environmental factors $[6,9-11]$. Sucrose, glucose and fructose are the major soluble sugars, and sucrose is the predominant sugar in melons at maturity being stored in the vacuoles of the pericarp parenchyma cells $[9,12]$. Both climacteric and nonclimacteric melons accumulate sugar during fruit ripening [6]. However, the sugar content of C. melo species differs according to the genetic variety and development stage $[9,13]$.
For example, the flexuosus melon group presents non-sweet and non-aromatic fruits, and the cantalupensis melon group has highly sweet and aromatic fruit [14]. Additionally, in fruit development, sugar is necessary for energy supply, it also generates turgor for fruit cell enlargement and accumulates in late stages of fruit (contributing to fruit taste) [15].

Sucrose accumulation in melon fruit is determined by the metabolism of carbohydrates in the fruit sink itself and can be provided from three main sources: (1) photosynthetic product; (2) raffinose family oligosaccharides (RFOs) catabolism; (3) sucrose resynthesis (Fig. 1). In sucrose accumulation, melon plants export sucrose, as well as raffinose family oligosaccharides (RFOs) such as raffinose and stachyose from photosynthetic sources (leaves) to sink tissues (developing melon fruit). RFOs are hydrolyzed by two different families of $\alpha$-galactosidase (neutral $\alpha$-galactosidase/NAG or acid $\alpha$-galactosidase/AAG) producing sucrose and galactose. The synthesized galactose is then phosphorylated by galactokinase (GK) and the resulting galactose 1-phosphate (gal1P) can either participate in the glycolysis pathway through the product glucose-6-phosphate or be used for sucrose synthesis. In sucrose synthesis, galactose 1-phosphate is transformed into glucose 1-phosphate (glc1P) by the actions of UDPgal/glc pyrophosphorylase (UGGP) and converted to other hexose-phosphates, providing the substrates for the synthesis of sucrose by sucrose-phosphate synthase (SPS) and sucrose-phosphate phosphatase (SPP). Furthermore, sucrose resynthesis is an important pathway and involves many enzymes of sugar

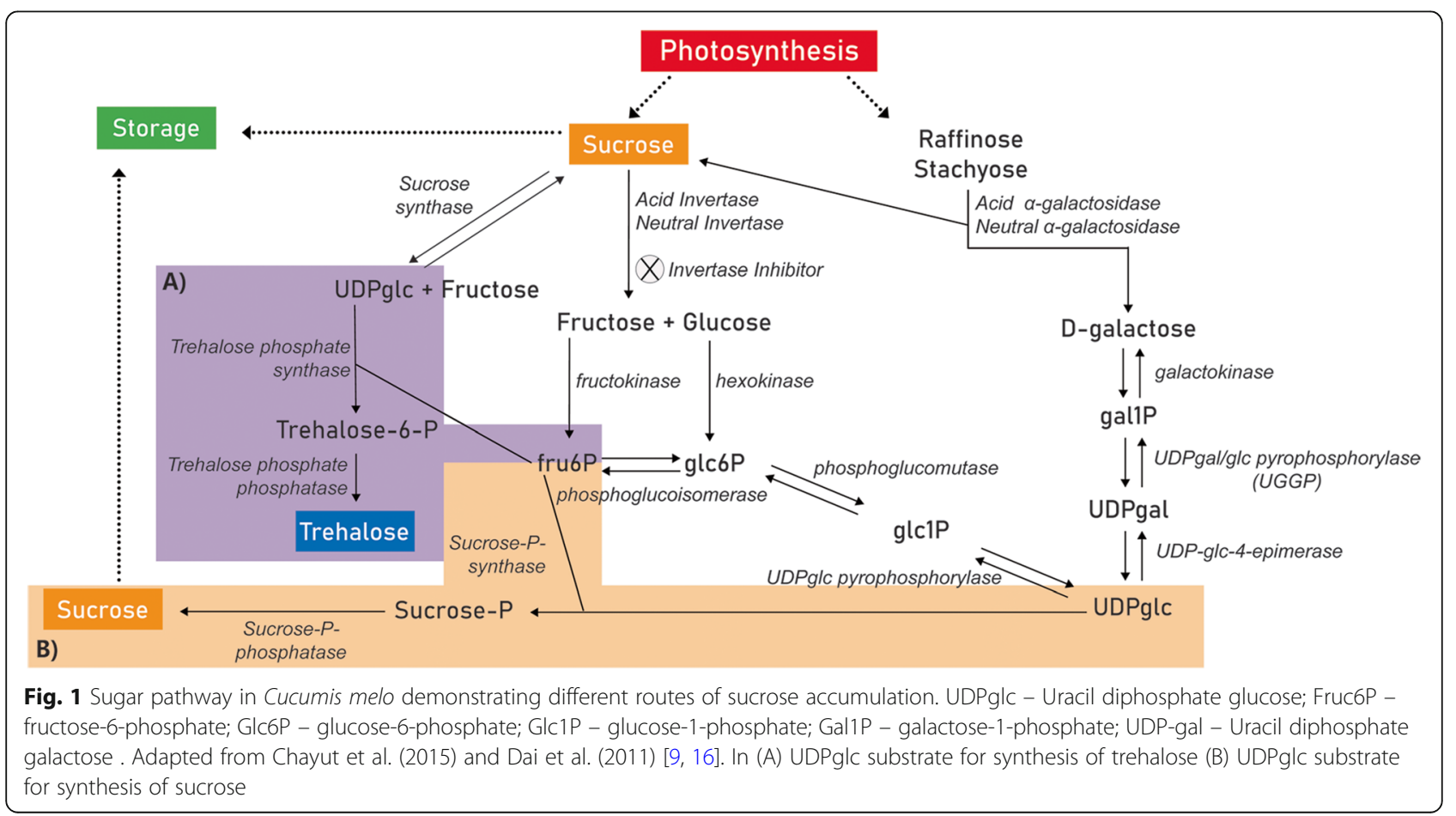


metabolism. On the afore-mentioned pathway, sucrose unloaded from the phloem can be hydrolyzed in the apoplast by cell wall invertases (CINs), however, in melon these enzymes may not have a crucial importance once cucurbits have a symplastic phloem loading. Then, the hexose sugar (glucose and fructose) products are imported into the cells by monosaccharide transporters, phosphorylated by hexokinase (HXK) and fructokinase (FK) and used for respiration or sucrose resynthesis. Within the cell, sucrose can be resynthesized in the cytosol by sucrose synthase (SUS) from fructose and UDP-glc. Sucrose can be hydrolyzed to fructose and glucose for energy production also by neutral invertase (NIN), or imported into the vacuole for storage or even hydrolyzed by vacuolar acid invertase (AIN). The invertase activity can be post-translationally regulated by invertase inhibitor proteins (INH) [3, 6, 9, 16-18].

The evidence that shelf life can be related to the sugar accumulation metabolism as well as the relevance of sugar content as a ripeness marker in non-climacteric melon, make sugar metabolism studies important to develop new approaches that can improve its commercial quality. Previous studies have elucidated the peculiarities of carbohydrate metabolism, mainly in climacteric melons $[3,9]$. However, understanding of the biochemical aspects that govern the different patterns of sucrose accumulation in the wide genetic variety of melon during the ripening process as well as the identification of new enzymes related to this pathway are limited. Comprehensive molecular studies that could enlighten the complexity of this metabolic pathway are essential. In the last decades, next-generation sequencing (NGS) or high-throughput techniques and metabolomic technologies allowed the generation of a vast amount of information that is essential for the global understanding of metabolic networks. Thus, the aim of our study was to comparatively analyze the transcriptomes of different development and ripening stages of non-climacteric 'Yellow' melon (Inodorus group) fruits focusing on the sugar pathway. Our analyses provide insights in gene expression ripening profiles of an important Brazilian commercial melon ranked in second position for the total amount of fruits exported by the country (197.60 million metric tons in 2018) [19].

\section{Results}

Variations in colour, pH and SS (soluble solids) during ripening of melon (Inodorus group)

Colour, $\mathrm{pH}$ and SS are important characteristics to determine the fruit development stage and changes in its chemical constituents. These parameters were evaluated on non-climacteric melon fruit of the 'Yellow' commercial genotype (Inodorus group) at 10 days after pollination (DAP), 20 DAP, 30 DAP and 40 DAP (Fig. 2a).
Colour measurement was expressed by the CIE (Commission Internationale de l'Eclairage) and Hue angle. Colorimeters express colours in numerical terms (see methods) along the $\mathrm{L}^{*}, \mathrm{a}^{*}$ and $\mathrm{b}^{*}$ axes (from white to black, green to red and blue to yellow, respectively) [20]. The results showed that $\mathrm{L}^{*}$ (brightness) of peel increases up to $20 \mathrm{DAP}$, declines up to $30 \mathrm{DAP}$ and remains stable until 40 DAP (Fig. 2b). For pulp colour, there was a decline in brightness until 30 DAPS with later stability (Fig. 2b). Coordinates on the a* axis increased during ripening for peel and pulp, representing the change from green to red (Fig. 2c). Coordinates on the $b^{*}$ axis increase during peel maturation demonstrating a shift from blue to yellow colour, that it is the opposite of the pulp profile (Fig. 2d). Hue angle $\left(\mathrm{H}^{\circ}\right)$ is variable as the true colour of the fruit and decreases with maturity, corroborating the findings of Kasim and Kasim (2014) [21] (Fig. 2e). The pH fruit showed a subtle increase during melon maturation (Table 1). Concerning soluble solids (SS) concentration, there is a gradual increase during the ripening process (Table 1).

\section{Transcriptome sequencing}

RNA-seq (RNA sequencing) was carried out on the complementary DNA libraries (cDNA) derived from 10 DAP (two biological replicates) and 40 DAP (three biological replicates) flesh mesocarp. The sequencing data were evaluated for quality, and were subject to data filtering. The results generated $\sim 59$ million clean single reads of $\sim 100 \mathrm{bp}$ in length. A total of $\sim 53$ million filtered reads were mapped to the Cucumis melo reference genome (https://www.melonomics.net) [22, 23] using Bowtie2 [24]. Most sample reads (79.65-97.88\%) were successfully aligned and for RNA-seq analysis, only the reads with overlapping in a single gene were considered (Table 2 and Additional file 1: Table S1).

\section{Ripening and development of fruit gene expression profile}

RNA-seq is an efficient and powerful tool for studying gene expression. The expression for each gene and differential expression (DE) analyses were calculated by statistical test evaluating the negative binomial distribution, being considered significant padj $\leq 0.05$ (see Methods). In this analysis, over 15,000 expressed genes were detected in each sample (Table 2 and Additional file 1: Table S2) of the 29,980 annotated in the Cucumis melo genome [22, 23]. However, a total of 1804 genes showed significant DE between the evaluated stages of fruit maturation (Additional file 1: Table S2). Of these, 895 were 10 DAP-biased and 909 were 40 DAP-biased as demonstrated in MA-plot (Additional file 1: Table S2 and Additional file 2: Figure S1). The RNA-seq data were validated by quantitative reverse transcription PCR analysis (RT-qPCR) of 8 transcripts in the 10 DAP and 40 DAP 


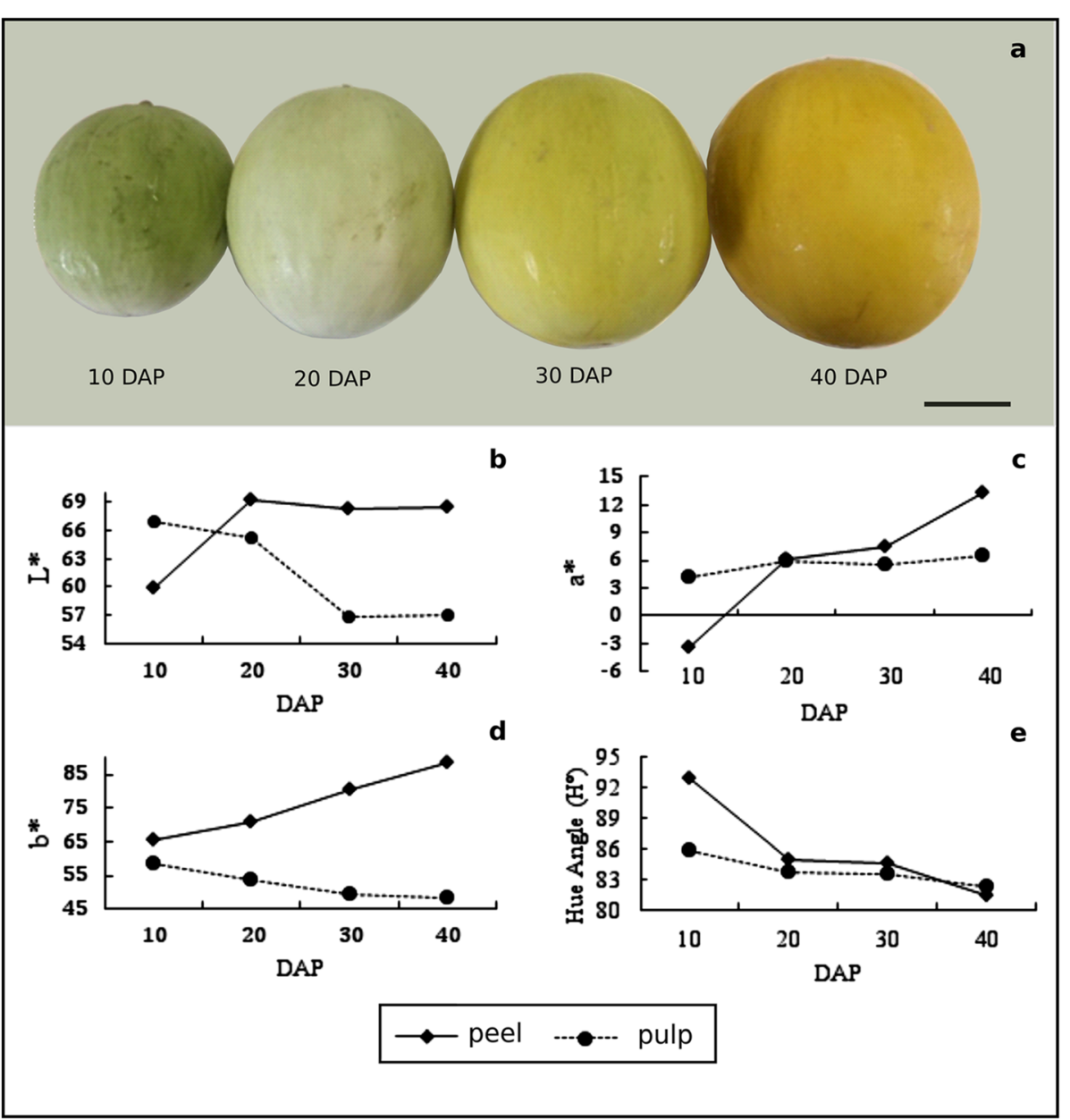

Fig. 2 Non-climacteric melon fruit of a 'Yellow' commercial genotype of four development stages and its colour characteristics. From left to right there are 10 DAP, 20 DAP, 30 DAP and 40 DAP (a). In (b) L* (brightness) of peel fruit increases from 10 to 20 DAP and declines in 30 DAP and remains constant until 40 DAP. In (c) the coordinates on the $a^{*}$ axis increase during the maturation process in peel and pulp colour, representing the trend change from green to red. In (d) coordinates on the $b$ * axis increase during maturation for peel colour, demonstrating the shift from blue to yellow coloration, the opposite profile was found for pulp colour. In (e) Hue angle $\left(\mathrm{H}^{\circ}\right)$ decreased throughout ripening, corroborating with Kasim and Kasim (2014)

melons (genes related to the sugar pathway), using CmRPS15 and CmRPL as reference genes (Fig. 3, Additional file 3: Table S3). From pairwise comparison of RNA-seq and RTqPCR analysis, Pearson's correlation coefficient was 0.98 $(p=0.0014)$ indicating positive correlation between the two methods (Additional file 3: Figure S2). The sample-to-sample distances that give an overview of similarities and dissimilarities between samples demonstrated clustering of young fruits (10 DAP) separately from the mature fruits (40 DAP)

Table $1 \mathrm{pH}$ and Soluble Solids (SS) ( ${ }^{\circ}$ Brix) mean for yellow melon (commercial cultivar) with 10 DAP, 20 DAP, 30 DAP. and 40 DAP

\begin{tabular}{lllll}
\hline & 10 D.A.P. & 20 D.A.P. & 30 D.A.P. & 40 D.A.P. \\
\hline pH & 4,15 & 4,7 & 4,85 & 5,1 \\
SS ('Brix) & 5,0 & 8,5 & 10,9 & 13,3 \\
\hline
\end{tabular}

(Additional file 4: Figure S3). Gene ontology (GO) enrichment analysis was performed using FDR (false discovery rate) adjusted $p$-value $<0.05$ on $\mathrm{DE}$ genes to characterize the differences of 'Yellow' melon development and ripening. Figure 4 and Additional File 5: Table S4 show the assigning of GO terms according to the equivalent biological process (BP), molecular function (MF) and cellular component (CC). We found that genes related to BP such as metabolic, physiological, transport and signalling processes were highly enriched in the 10 DAP stage DE genes. On the other hand, $\mathrm{DE}$ genes of the 40 DAP fruit were more abundant in the cellular process, cellular nitrogen compound and peptide metabolism BP categories. Under the cellular component classification, the DE genes of the young fruit were only significantly enriched within the 'membrane' category, while $\mathrm{DE}$ genes of the mature fruit were enriched in several CC terms (e. g. 'cytoplasm', 'chloroplast, ribosomes'). The top 3 
Table 2 Number of filtered reads from each sample sequenced and mapped to the Cucumis melo (https://www.melonomics.net) reference genome

\begin{tabular}{lllll}
\hline Sample name & Input reads (filtered) & Mapped reads & \% of mapped reads & Detected genes \\
\hline 10DAP_V2 & $13,444,823$ & $13,159,483$ & $97.88 \%$ & 16,865 \\
10DAP_V3 & $11,805,793$ & $10,577,826$ & $89.60 \%$ & 16,756 \\
40DAP_M1 & $11,591,063$ & $10,113,177$ & $87.25 \%$ & 16,161 \\
40DAP_M2 & $12,635,568$ & $11,246,225$ & $89.00 \%$ & 15,975 \\
40DAP_M3 & $10,203,078$ & $8,127,127$ & $79.65 \%$ & 15,090 \\
\hline
\end{tabular}

groups within the MF classification were 'catalytic activity', 'ion binding' and 'hydrolase activity' for the 10 DAP stage; and 'binding, structural molecule activity' and 'structural constituent of ribosome' for the 40 DAP stage.

Hierarchical clustering was performed on the 50 most significant DE genes of the 10 DAP and 40 DAP fruits. The clustering of genes was represented in a heatmap (Additional file 6: Table S5 and Figure S4). The results showed 2 genes involved in 'starch and sucrose metabolism' (cmo0500) that were more expressed in 10 DAP fruit (beta-glucosidase and sucrose synthase 2); and 2 related to 'hormone signal transduction' (cmo04075), being 2 genes more expressed in 10 DAP fruit (xyloglucan endotransglucosylase/hydrolase) and 1 gene more expressed in 40 DAP (pathogenesis-related protein 1-like).

\section{KEGG enrichment analyses and network construction}

The RNA-seq results were subjected to a KEGG pathway enrichment analysis (DAVID software [26]) to elucidate the main pathways involved in fruit ripening and development. A total of $92 \%(1668 / 1804)$ of the DE genes could be converted into UniProtID (available in the
DAVID software database). Table 3 shows the top 6 most significantly enriched KEGG pathways for both development stages. The young fruit was enriched with 'plant hormone signal transduction' and energetics metabolisms including 'starch and sucrose metabolism'. The full-ripe melon presented more genes involved with 'protein processing in endoplasmic reticulum' and 'spliceosome', in addition to energetic metabolisms (Additional File 7: Table S6). Interestingly, the ethylene receptor 1 (MELO3C003906.2) that is a gene of ethylene hormone signal transduction was more expressed in 40 DAP than 10 DAP (Table 4, Additional File 7: Table S6). In this study, we focused on sucrose metabolism (related routes were also considered) because this is an important pathway associated with fruit quality traits. The other pathways will be analyzed in more detail in further studies.

For network construction, we used the STRING database (https://string-db.org) that returned 417 nodes, 671 edges and the $p$-value for protein-protein interaction (PPI) enrichment was < 1.0e-16 for 10 DAP fruit genes (Additional file 8: Figure S5, Table S7). The 40 DAP fruit genes results showed 404 nodes, 1512 edges and the p-value PPI enrichment was
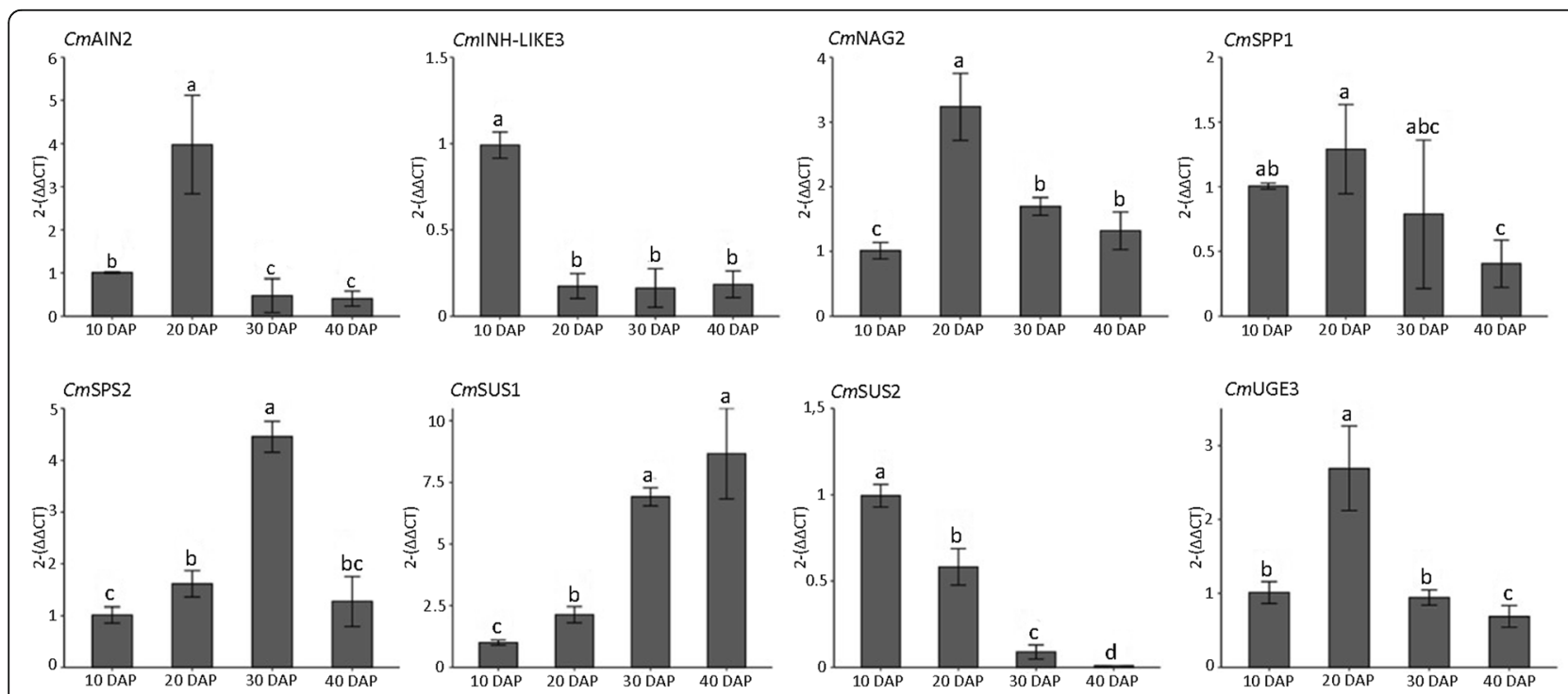

Fig. 3 The relative mRNA expression of 9 genes of the sucrose metabolism was determined by $2^{-\Delta \Delta C t}$ [25]. Results are expressed as mean \pm SEM and significance of different developmental stages (10 DAP, 20 DAP, 30 DAP, 40 DAP) comparison is defined as $p \leq 0.05$ by Tuckey test after data normalization by Box-Cox method or by Kruskal-Wallis \& Wilcoxon (CmSUS1 and CmSUS2). Different letters indicate significant differences 


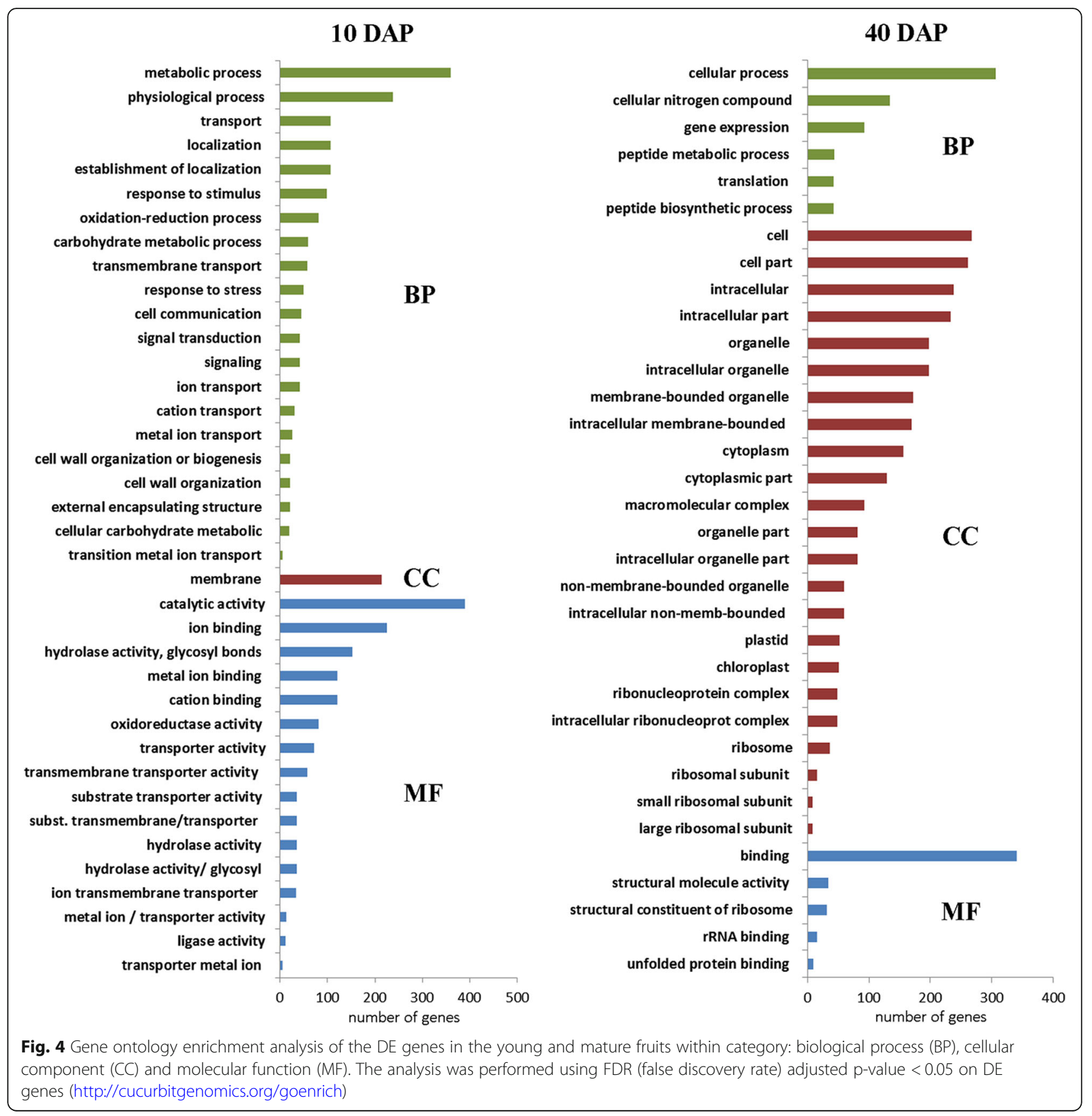

$<5.79 \mathrm{e}-08$ (Additional file 8: Figure S6, Table S8). The functional enrichment in the network demonstrated a high number of proteins involved in metabolic pathways and protein processing in the young and full-ripe fruit respectively (Additional file 8: Figure S5, S6). Proteins related to the sugar pathway were selected from total DE genes and the subnetwork generated was composed of 38 nodes, 68 edges and PPI enrichment p-value $<1.0 \mathrm{e}-16$ in young fruit. The proteins with the highest interaction in this analysis were alpha-N-arabinofuranosidase 1 (XP_008443206.1), sucrose synthase (XP_008463167.1) and acid invertase 2 (NP_
001284469.1) (Fig. 5, Additional file 9: Tables S9, S10). Regarding the mature fruit, the subnetwork generated was characterized by 22 nodes, 27 edges and PPI enrichment pvalue $<1.0 \mathrm{e}-16$. The protein argonaute 1 (XP_008438929.1) and probable galacturonosyltransferase 10 (XP_008447733.1) presented the highest interactions number (Fig. 5, Additional file 9: Tables S11, S12).

\section{Sugar pathway and associated proteins}

Seventeen DE genes are associated with the sucrose metabolism by KEGG analyses (Fig. 6, Table 4, Additional file 10: 
Table 3 KEGG pathway analysis of fruit ripening and development candidates genes

\begin{tabular}{|c|c|c|c|}
\hline \multicolumn{4}{|l|}{10 DAP fruit } \\
\hline KEGG pathway & Gene count & $\%$ & Fisher Exact $\boldsymbol{P}$-value \\
\hline $\begin{array}{l}1 \text { Plant hormone signal } \\
\text { transduction }\end{array}$ & 21 & 2.5 & $4.3 \mathrm{E}-3$ \\
\hline 2 Carbon metabolism & 16 & 1.9 & $6.7 \mathrm{E}-2$ \\
\hline $\begin{array}{l}3 \text { Starch and sucrose } \\
\text { metabolism }\end{array}$ & 11 & 1.3 & $5.7 \mathrm{E}-3$ \\
\hline 4 Photosynthesis & 7 & 0.8 & $2.8 \mathrm{E}-3$ \\
\hline 5 Galactose metabolism & 7 & 0.8 & $1.1 \mathrm{E}-2$ \\
\hline $\begin{array}{l}6 \text { Carbon fixation in } \\
\text { photosynthetic organisms }\end{array}$ & 6 & 0.7 & $7.5 \mathrm{E}-2$ \\
\hline \multicolumn{4}{|l|}{40 DAP fruit } \\
\hline KEGG pathway & Gene count & $\%$ & Fisher Exact $P$-value* \\
\hline $\begin{array}{l}1 \text { Protein processing in } \\
\text { endoplasmic reticulum }\end{array}$ & 24 & 2.8 & $5.5 \mathrm{E}-7$ \\
\hline 2 Spliceosome & 17 & 2 & $4.0 \mathrm{E}-4$ \\
\hline 3 Carbon metabolism & 16 & 1.9 & $5.0 \mathrm{E}-2$ \\
\hline $\begin{array}{l}4 \text { Ribosome biogenesis in } \\
\text { eukaryotes }\end{array}$ & 11 & 1.3 & $4.8 \mathrm{E}-4$ \\
\hline $\begin{array}{l}5 \text { Carbon fixation in } \\
\text { photosynthetic organisms }\end{array}$ & 7 & 0.8 & $2.3 \mathrm{E}-2$ \\
\hline 6 Pyruvate metabolism & 7 & 0.8 & $4.9 \mathrm{E}-2$ \\
\hline
\end{tabular}

* Significant P-value $\leq 0.05$

Figure S7, Additional file 11: Figure S10). The genes that present higher interaction with these enzymes (STRING database) by PPI analyses and those that are important in sucrose metabolism described in previous studies (not available in the KEGG database) were also considered [9, 16] (Fig. 6, Table 4, Additional file 10: Figures S8, S9, Additional file 11: Figure S10). Some enzymes associated with this pathway are encoded by multiple genes and their amino acid sequences were aligned using the MUSCLE algorithm [27] as well as submitted to percentage similarity analysis (http://imed.med.ucm.es/Tools/sias.html software). The results showed a wide difference between the isoenzymes; and the alpha galactosidases, invertase inhibitor and hexosyltransferase sequences were the most dissimilar (Additional file 12: Figure S11).

Considering RNA-seq analysis, in the 'starch and sucrose metabolism' (KEGG: cmo00500) 12 genes are more expressed in young fruit (acid invertase 2/ CmAIN2, phosphoglucomutase/CmPGIcyt, alpha-amylase/CmAAML, alpha-trehalose-phosphate synthase9/CmTPS9, betaglucosidase 18-like/CmBGL18, beta-glucosidase 24/ $C m B G L 24$, endoglucanase-like/CmEGLC, inactive betaamylase/CmIBAML, sucrose synthase 2/CmSUS2, sucrose-phosphatase1/CmSPP1, sucrose-phosphate synthase $2 / C m S P S 2$, trehalose 6 -phosphate phosphatase $1 /$ CmTPP1); and 5 genes are more expressed in full-ripe fruit (beta-amylase/CmBAML, glucan endo-1,3-beta- glucosidase 1/CmGBGL1, sucrose synthase 1/CmSUS1, trehalose-6-phosphate synthase 7/CmTPS7, trehalose-6phosphate synthase 5/CmTPS5) (Table 4, Additional file 11: Figure S10). The highest log2 fold change values were to CmEGLC (7.8063) and CmBGL24 (4.5276) in young melon. For mature melon they were to CmGBGL1 (1.8416) and CmSUS1 (1.2647) (Table 4). The RT-qPCR (quantitative reverse transcription PCR analysis) was conducted for some of these genes in the 10 DAP, 20 DAP, 30 DAP and 40 DAP stages (Fig. 3). In this analysis, the CmAIN2 gene has a markedly increased expression from 10 to 20 DAP fruit, declining rapidly in subsequent stages (Fig. 3). The two sucrose synthase isoenzymes showed different expression patterns in fruit maturation as also observed in RNA-seq. CmSUS1 relative expression has a continuous increase from 10 DAP to 40 DAP fruit. In contrast, the CmSUS2 gene has a higher expression level in younger fruit and gradually decreased in the following ripening stages (Fig. 3). The expression level of CmSPS2 was more remarkable in 30 DAP fruits when compared to other maturation stages (Fig. 3). CmSPP1 expression increased from 10 DAP to 20 DAP and then decreased in the following developmental stages (Fig. 3). The CmINH-LIKE3 is not presented in the KEGG pathway; however, it has been included in RT-qPCR analyses because the literature reports its function in invertase inhibition. The expression profile of this gene demonstrated a marked expression only in younger fruit when compared to other development stages (Fig. 3). However, the CmINH2 isoform presented higher expression in 40 DAP fruit when compared to 10 DAP fruit (RNA-seq analysis).

In the 'amino sugar and nucleotide sugar metabolism' (cmo00520), 7 genes are more expressed in 10 DAP fruit (UDP-glucose 6-dehydrogenase/CmUG6D, Acidic endochitinase/CmAEChit, Alpha-L-arabinofuranosidase 1-like isoform/CmALAR, Endochitinase EP3-like/CmEP3-Like, Hevamine-A-like/CmHV-ALIKE, Hexosyltransferase 3/ CmHEXT3, UDP-glucose epimerase 3/CmUGE3) and 1 gene is more expressed in 40 DAP (UDP-sugar pyrophosphorylase/CmUGGP) (Table 4, Additional file 11: Figure $\mathrm{S} 10)$. The most representative expression level was to $C m H V$-ALIKE (4.4816). In the RT-qPCR analysis, the gene expression of $C m U G E 3$ was relatively low in young fruit, increased rapidly in the 20 DAP stage and decreased in the following developmental stages (Fig. 3).

The 'galactose metabolism' (cmo00052) has 9 DE genes, 6 of them more expressed in young fruit (Alkaline alphagalactosidase/CmNAG2, Alpha-galactosidase 2/CmAAG2, Galactinol-sucrose galactosyltransferase 5/CmNAGLIKE2, Stachyose synthase/CmSCS, Acid Invertase 2/CmAIN2, Phosphoglucomutase/CmPGIcyt) and 3 more expressed in mature fruit (UDP-sugar pyrophosphorylase/CmUGGP, ATP-dependent 6-phosphofructokinase/CmATP-PPKN, Galactinol-sucrose galactosyltransferase 6 isoform X1/ 


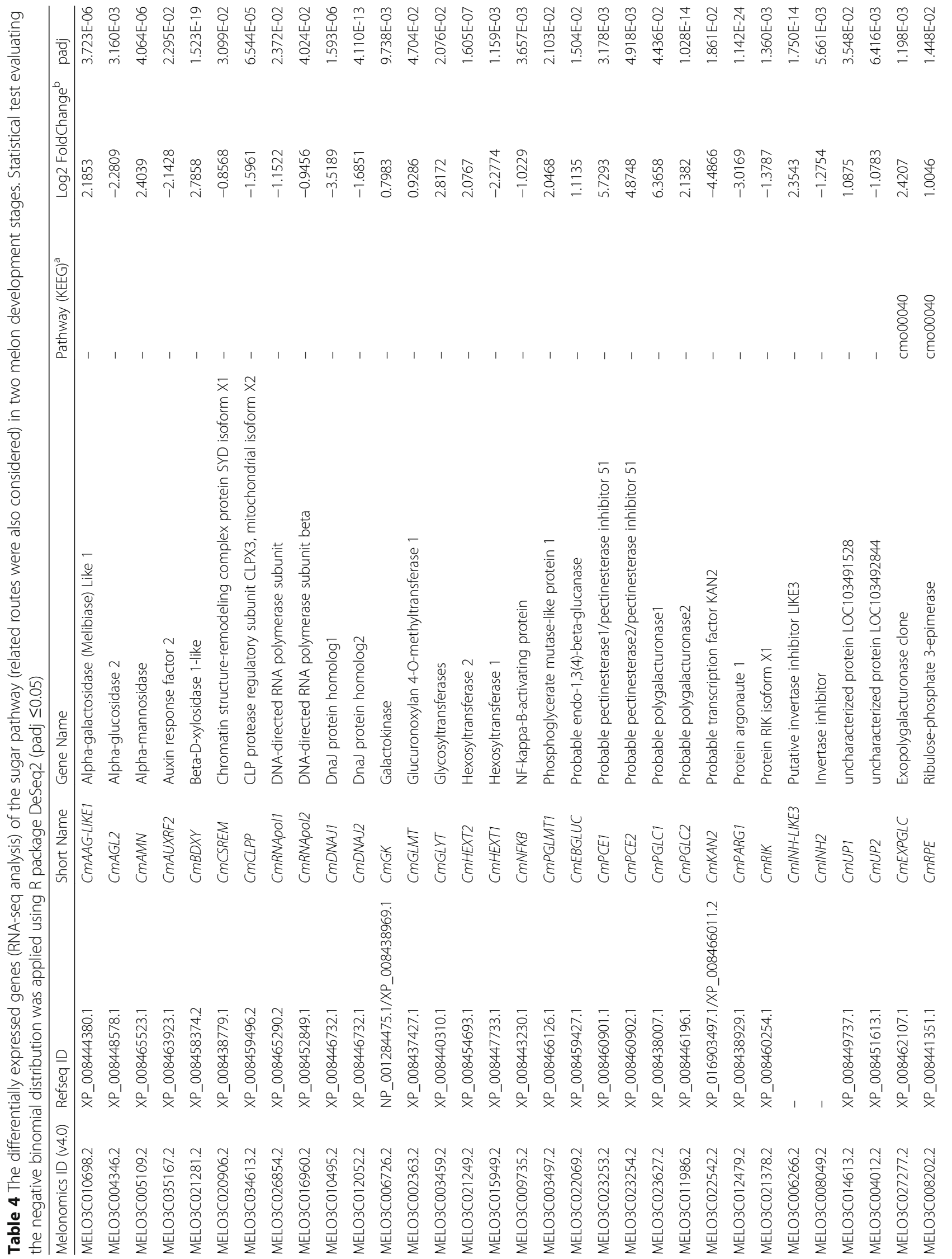




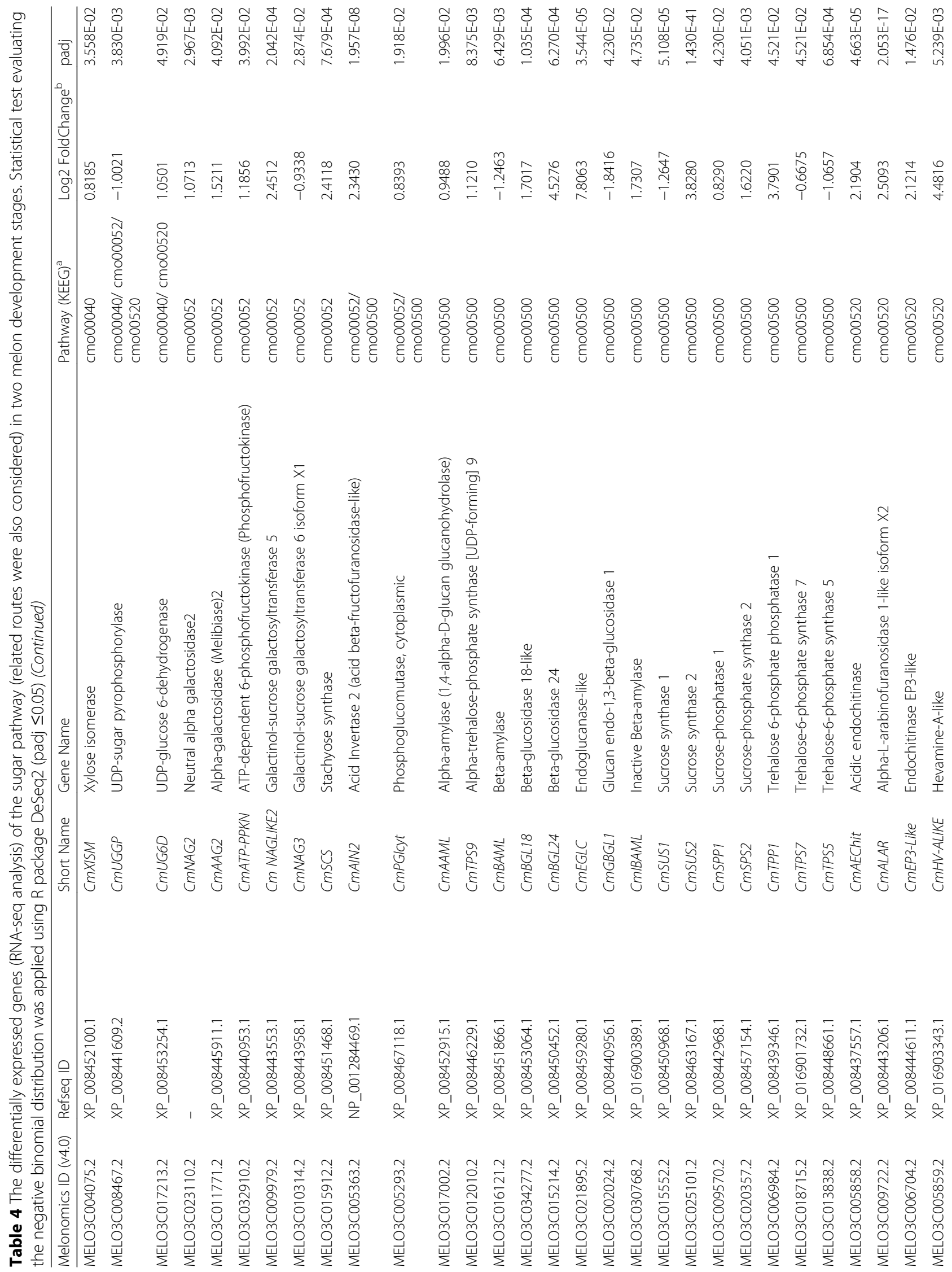




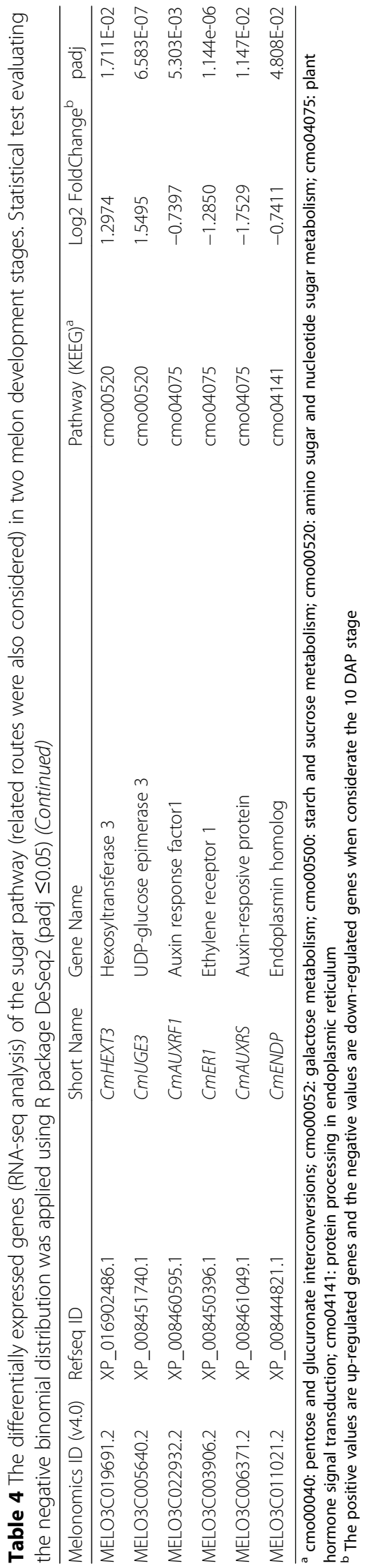




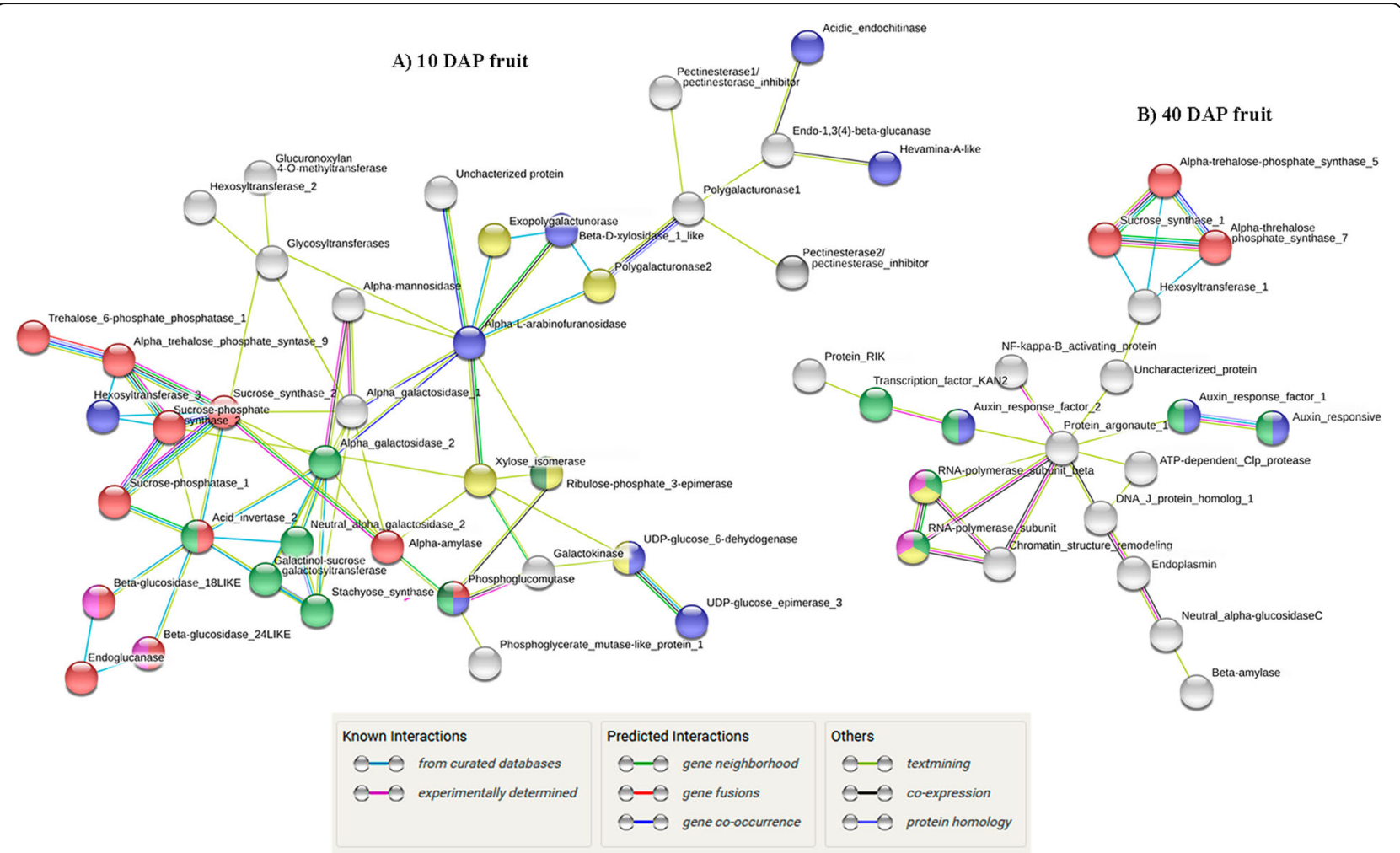

Fig. 5 Protein-protein interaction network of sugar pathway and associated routes in the 10 DAP (a) and 40 DAP (b) melon fruits obtained by STRING analyses. Nodes represent related proteins and edges represent protein-protein associations. In A) red is "Starch and sucrose metabolism", blue is "Amino and nucleotide sugar metabolism", light green is "Galactose metabolism", yellow is "Pentose and glucoronate interconversions", pink is "Cyanoamino acid metabolism" and dark green is "Pentose phosphate pathway". In B) red is "Starch and sucrose metabolism", blue is "Auxin signalling pathway", green is "Transcription", yellow is "Nucleotidyltransferase" and pink is "DNA-directed RNA polymerase". The white nodes are genes not classified within a pathway or protein group

CmNAG3) (Table 4, Additional file 11: Figure S10). The relative expression of the $C m N A G 2$ gene showed a rapid increase from 10 DAP to 20 DAP decreasing in 30 DAP and keeping constant in 40 DAP (Fig. 3). The RT-qPCR of CmAIN2 has been previously discussed.

Also, another 32 DEGs were identified in network analyses that are potentially associated with the sugar pathway (Table 4, Additional file 11: Figure S10). The more expressed genes were: probable polygalacturonase1 (6.3658), probable pectinesterase1 (5.7293), probable pectinesterase 2 (4.8748) for young fruits and probable transcription factor KAN2 (4.48), DnaJ protein homolog1 (3.51), Protein argonaute 1 (3.016) for full-ripe fruits.

\section{Discussion}

\section{Global characteristics of the 'yellow' non-climacteric melon ripening}

Fruit ripening and development is a genetically programmed and irreversible process that involves physiological, biochemical and organoleptic changes influencing the fruit quality such as flavour, texture, colour and aroma [28]. However, the study of the metabolic networks is complex and the central signal of genic cascade is not completely understood. In our study, we used an important commercial non-climacteric 'Yellow' melon fruit (Cucumis melo, inodorus group) as experimental material to comprehend the main metabolic processes that involve maturation in this phenotype, focusing on the sugar pathway study that is a main quality attribute in melon fruits.

RNA-seq technology was used to analyze the transcriptomic differences between young (10 DAP) and mature (40 DAP) non-climacteric melon fruit. A total of $895 \mathrm{DE}$ genes are down-regulated and 909 are upregulated during melon ripening. $\mathrm{GO}$ enrichment analysis showed that the DE genes in young fruit were more related to molecular transport and metabolic processes including the 'carbohydrate metabolism'; while in ripe fruit the most DE genes are required for peptide metabolism and protein biosynthesis. In addition, the integrative KEGG analysis conducted for metabolic pathways demonstrated that 'carbon fixation in photosynthetic organisms' and 'carbon metabolism' pathways were enriched in both fruit development stages; however different genes or isoforms are DE. At the beginning of fruit development there is high anabolism and catabolism of sugar that is the metabolic process required for 


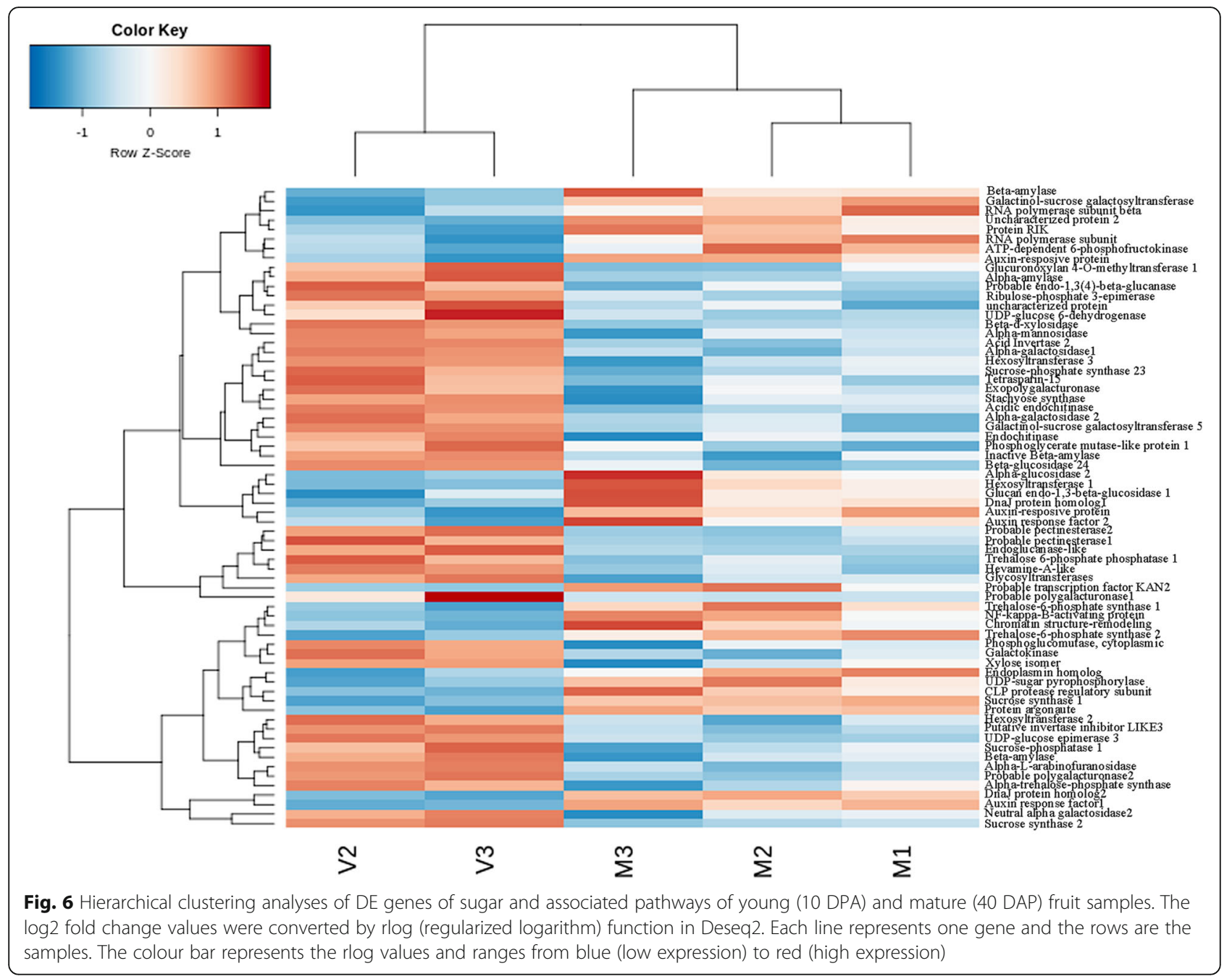

carbon skeleton construction and energy supply in plants. In strawberry fruit, an important role of oxidative phosphorylation in ripening was demonstrated [29]. The DE genes enriched in 40 DAP melon fruits are related to the sucrose accumulation function [6]. The protein processes in the endoplasmic reticulum, spliceosome mechanism and ribosome biogenesis were also significantly enriched in KEGG analysis in the late development of melon indicating high transcription and translation rate. Moreover, the high splicing process is reflected in the production of different proteins that can act and control a specific metabolic route. This characteristic associated with the activation of different protein isoforms can also explain the KEGG enrichment of the same pathways in both maturation stages as previously mentioned. Studies have reported the presence of paralogous copies acting in diverse metabolic pathways in plants, including in sugar metabolism, that in melon have definite functionalization concerning both development stages and tissue specificity [9]. The high activity of photosynthesis in young melon when compared to full-ripe fruit has also been described for grape and other melon varieties $[6,30]$.

The 'plant hormone signal transduction' is an important process in fruit ripening [31,32], and this pathway was significantly enriched in the early melon fruit development. Ethylene (ETH), abscisic acid (ABA) and brassinosteroids (BRs) have been suggested to promote ripening through complex interactions; while auxin (IAA), cytokinins (CYT), gibberellin (GA) and jasmonic acid (JA) are putative inhibitors of ripening [29,33]. In our study, the DE genes present in IAA, JA, GA and CYT signal transduction decreased during maturation which also occurs in other non-climacteric fruits $[29,34,35]$. In the ABA pathway, only the 'protein phosphatase $2 \mathrm{C} 77$ ' gene (repressor of the abscisic acid signalling pathway [36]) was DE in the 10 DAP fruit. Studies have been suggested that ABA plays an important role in the regulation of non-climacteric fruits $[37,38]$ and the key gene for its biosynthesis is 9-cisepoxycarotenoid dioxygenase (CmNCED) that was significantly more expressed in full-ripe fruit (Additional file 7 : 
Table S6). This can indicate that ABA might be involved in the regulation of melon maturation and senescence. Interestingly, there are intimate connections between sugar and ABA signalling [39]. The BR burst production generally occurs in the colour change stage in late fruit development $[29,33,40]$. In our study, the genes related to BR signal transduction are more expressed in young 'Yellow' melon fruit; however the colour change occurs from 20 DAP to 30 DAP fruits. Thus further studies should be conducted to understand the transcriptome profile of these stages. The expression of some genes present in the ethylene and salicylic acid metabolism were highest in mature fruit (Additional file 7: Table S6). One of these genes is the ethylene receptor 1 that has been shown to negatively regulate ethylene signal transduction and suppress ethylene responses [41]. Thus, it can be a candidate gene in nonclimacteric and climacteric melon comparative study.

In the subnetwork protein-protein interaction (PPI), the results of the 10 DAP fruits showed the interaction of 6 metabolic pathways: 'Starch and sucrose metabolism'; 'Amino and nucleotide sugar metabolism'; 'Galactose metabolism'; 'Pentose and glucuronate interconversions'; 'Cynoamino acid metabolism'; and 'Pentose phosphate pathway'. Furthermore, enzymes related to cell wall degradation were identified such as pectinesterase and polygalacturonase that are mainly responsible for the pectin changes. The up-regulation of these genes and those associated with sucrose synthesis in the early stage of development are involved with progressive fruit softening and sucrose accumulation. In flesh watermelon, some isoforms of pectinesterase and polygalacturonase also show an increase in the first development stages, decreasing in the full-ripe fruit [42]. Another cell wall enzyme was alpha-L-arabinofuranosidase that catalyzes the breaks in the arabinoxylan (major component of cell wall plant hemicellulose) [43]. Saladié et al. (2015) demonstrated that several genes related to cell wall degradation were more strongly up-regulated in climacteric melon (cv. Védrantais) than non-climacteric (cv. Piel de Sapo) [6]. The sugar metabolism is an important process in fruit ripening and development and sucrose accumulation is the major determinant of melon sweetness $[6,44]$. One enzyme of this pathway is the acid invertase (CmAIN2) that had the second highest number of interactions in the young melon fruit subnetwork (Fig. 5; Additional file 9: Table S9). This reinforces the idea of its key function in the catabolism of sucrose [6]. Two beta-glucosidases (CmGL18, CmGL24) have an interaction with $C m A I N 2$, these enzymes have the function of hydrolyzing the terminal, non-reducing betaD-glucosyl residues (final reaction in cellulose hydrolysis) with the release of beta-D-glucose (primary energy source in plants) [45] that suggest a high sugar conversion to energy in the early fruit development stage. Another important enzyme in the subnetwork is sucrose synthase
2 (CmSUS2) that has a strong interaction with alphatrehalose phosphate synthase 9 (CmTPS9) followed by trehalose phosphate phosphatase (CmTPP1). These enzymes and others of sugar metabolism will be discussed in more detail below in the next topic.

Although the majority of DE genes of auxin and sugar metabolism are up-regulated in 10 DAP melons, some isoforms or different genes from these pathways are more expressed in 40 DAP. The subnetwork generated for mature fruit is represented by a different sucrose synthase (CmSUS1) which also has high interaction with two alpha-trehalose phosphate synthase isoenzymes (CmTPS7, CmTPS5). The trehalose phosphate synthases (TPS) convert glucose-6-phosphate and uridine diphosphate (UDP) glucose into trehalose-6phosphate (T6P) and the subsequent dephosphorization of T6P is catalyzed by trehalose-phosphate phosphatases. A recent study reported that threalose-6-phosphate inhibited sucrose synthase and consequently the sucrose cleavage in castor bean [46]. The T6P may be undergoing a higher conversion into trehalose in young melon due to the greater trehalose-phosphate phosphatase gene expression. Thus T6P accumulation is expected in full-ripe fruit, once that TPP is down-regulated, contributing to the increase of sucrose content [47]. In addition, genes involved with the auxin pathway such as auxin response factor (CmAUXRF1, CmAUXRF2) and responsive auxin protein $(C m A U X R S)$ are present in this subnetwork and have interaction through hexosyltransferase and argonaute proteins with the CmSUS1, CmTPS7 and CmTPS5 (Fig. 5). In that respect, previous studies reported that auxin reduces the sugar content in fruits [48]. However, the precise association of the genes CmAUXRF1, $C m A U X R F 2$ and $C m A U X R S$ with the sugar pathway requires further studies. Regarding the argonaute proteins, they bind to micro RNAs (miRNA) and act in transcript cleavage [49]. Plant miRNAs typically target transcription factors including the auxin-response factor [49]. A weak interaction was detected between hexosyltransferases, unknown proteins and argonaute proteins and further studies should be conducted to better understand this association. It is also noteworthy that the chromatin structure-remodelling complex protein SYD $(C m C S R E M)$ gene present in this subnetwork is related to a promotor regulation of several genes downstream of the jasmonate and ethylene signalling pathways [50].

\section{Sucrose metabolism}

Sugar metabolism is an important pathway related to the sweetness of fruits and it is the most attractive characteristic for consumers [51]. Furthermore, studies have reported that sugars may serve as important signals that modulate a wide range of processes in plant physiology including fruit maturation $[39,52,53]$. In our study, a 
total of 17 genes were DEs in the sucrose; amino and nucleotide sugar; and galactosidase pathways and 8 were evaluated in two additional development stages (20 and 30 DAP). Sucrose is the main sugar component that gives the sweet taste in melon and its high content at the mature stage could be used as a marker [6]. Only sucrose synthases and invertases are known enzymes responsible for sucrose cleavage [10]. The sucrose synthases convert sucrose to fructose and UDP glucose that is a reversible reaction [10]. In our study, two isoforms were DEs by RNA-seq analysis, the CmSUS1 that was up-regulated in full-ripe fruit while CmSUS2 had a burst of gene expression in 10 DAP fruit. In fruit maturation, there is a gradual expression increase of CmSUS1 and decrease of CmSUS2 in the 'Yellow' melon. In non-climacteric melons, 'Hami' [51] and 'Piel del Sapo' [6], the same expression profile was observed. In 'Dulce' climacteric melon, the CmSUS1 was more expressed in young fruit, followed by near-silencing in mature fruit. CmSUS2 showed low levels of expression throughout fruit development and the third sucrose synthase (CmSUS3) was DE being weakly expressed in the young fruit and increased in the maturing fruit [9]. Thus, it may be suggested that in non-climacteric melons, CmSUS1 is mainly responsible for the synthesis of sucrose for storage in the vacuole, contributing to ripe fruit taste, while CmSUS2 acts in an opposite way providing the substrate for energy production by sucrose catabolism during early development (Fig. 7). Also, the TPS and TPP have an important function in the sucrose synthase activities contributing to sucrose content in the fruit as previously described (Fig. 7).

Invertases produce glucose instead of UDP-glucose and fructose in a non-reversible reaction. Acid invertases have been attributed to vacuole localization while neutral invertases have generally been located in the cytosol, consistent with the optimal neutral $\mathrm{pH}$ activity and absence of glycosylation [9]. In the RNA-seq analysis, only the acid invertase (CmAIN2) was DE in non-climacteric 'Yellow' melon. Previous studies with 'Piel del Sapo' [6] and 'Hami' non-climacteric melon fruit [51] also showed only transcriptional activity of acid invertase 2 (CmAIN2). In 'Dulce' climacteric melons, four neutral invertase (CmNIN1, CmNIN2, CmNIN3 and CmNIN4) were DE, as well as the acid invertase 2 (CmAIN2) [9]. The peak of CmAIN2 expression occurs in the 20 DAP 'Yellow' melon fruits and consistently decreased in the following developmental stages (Fig. 7). Studies have demonstrated that acid and neutral invertase genes are highly expressed in young developing fruit, and subsequently declined substantially at the sucrose accumulation stage $[6,9,16,51]$. This reduction of soluble acid invertase activity signals the metabolic transition from fruit growth to sucrose accumulation $[3,18]$. The higher expression of neutral invertases in climacteric melon fruit suggests that cytoplasmatic sugar catabolism might be an additional source of energy, supporting the hypothesis that climacteric melon fruit spends more energy during fruit development, due to respiration, than nonclimacteric ones. In the non-climacteric and climacteric

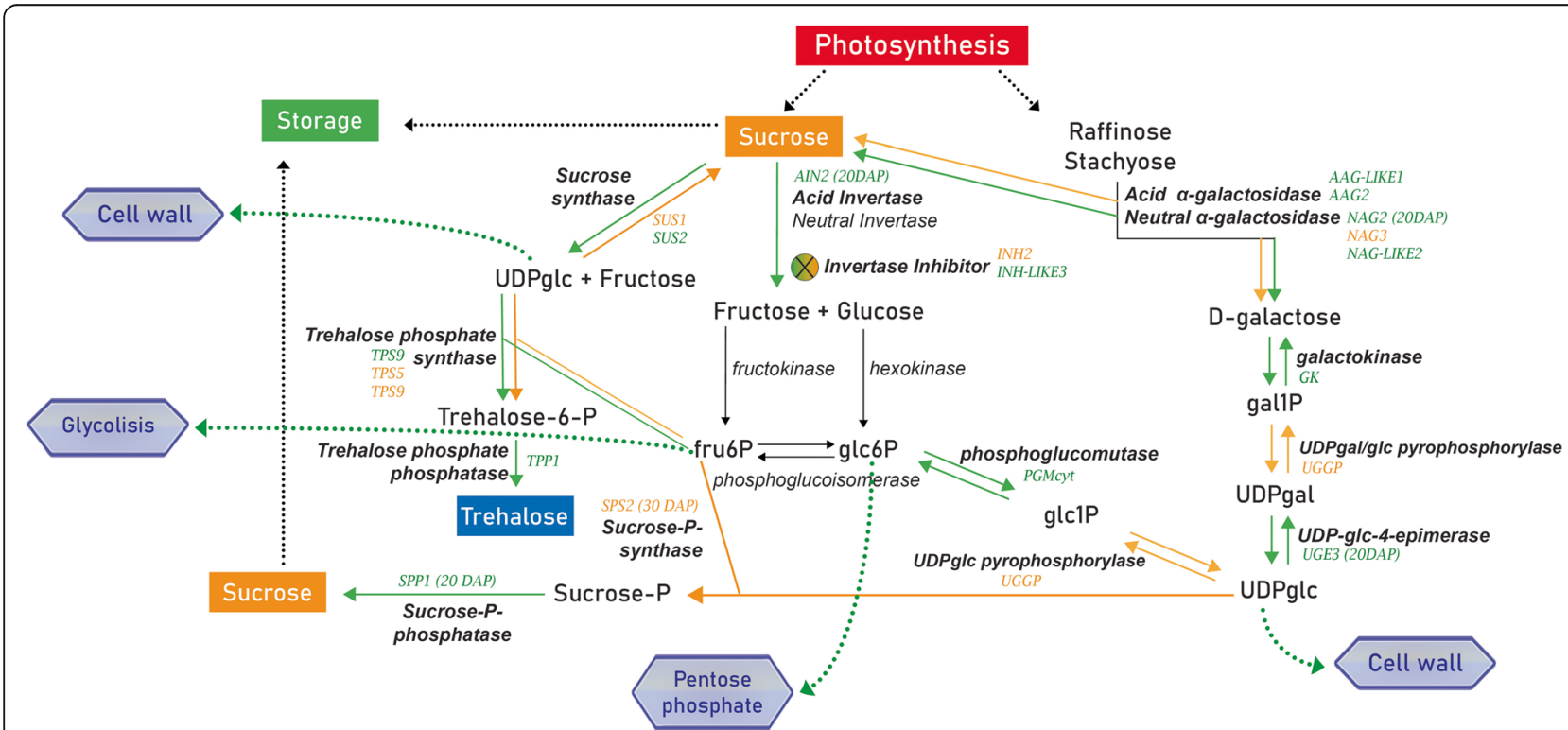

Fig. 7 Differential expression of sugar metabolism related genes in the melon ripening process. The green arrows represent 10 DAP or 20 DAP fruits and the yellow arrows 30 DAP or 40 DAP fruits. The genes with burst of expression in the intermediate stages (20 DAP and 30 DAP) have the phase indicated in parentheses 
melon comparison, studies demonstrated that the acid invertase gene (CmAIN2) was almost 10-fold higher in 'Védrantais' (climacteric) than in 'Piel del Sapo' (non-climacteric). The high activity of soluble acid invertase (CmAIN2) might limit the accumulation of sucrose during climacteric ripening and increase organic acids, such as malate, that impart a stale flavour to the fruit $[3,6,9,18]$. Thus, the inhibition of CmAIN2 can be a key process in the differences of sugar content and melon quality.

Invertase inhibitors are responsible for decreasing the activity of soluble acid invertases through post-translational regulation, reducing sugar consumed in respiration and regulating the accumulation of sucrose during melon development and ripening $[6,18]$. Two invertase inhibitors (CmINH2; CmINH-LIKE3) were DE by RNA-seq analysis and are characterized by the presence of plant invertase/ pectin methyltransferase inhibitor domain (Additional file 12: Figure S11). The invertase inhibitor 2 presented higher transcription level in full-ripe (40 DAP) than in youngest melon (10 DAP) (Fig. 7). On the other hand the putative invertase inhibitor 3 (CmINH-LIKE3) had high activity in the beginning of development and low expression in the following stages (Fig. 7). Hence, in the 20 DAP stage the inactivation of CmAIN2 by CmINH-LIKE3 protein interaction may be occurring. Subsequently, the CmAIN2 expression rapidly decreases and the CmINH-LIKE3 transcription is not necessary anymore. The $\mathrm{CmINH} 2$ can have affinity with other invertases that have not been evaluated on the intermediate stages (20 DAP and 30 DAP). The putative CmINH-LIKE3 expression was not reported in previous studies. The DE of $\mathrm{CmINH} 2$ was also detected in climacteric 'Dulce' melon; however, it is more strongly expressed in the first stages of development decreasing during ripening [9]. One other $\mathrm{DE}$ isoform detected for this same variety was $\mathrm{CmINH1}$ that was expressed at high levels in the 30 DAP stage [9]. The results demonstrated different expression profiles for non-climacteric 'Yellow' melon and climacteric 'Dulce' melon, indicating recruitment of distinct $\mathrm{INH}$ isoforms in the regulation of the invertases or its activation in different stages of ripening. Moreover, two invertase inhibitors (cCL2226Contig1 and c15d_02-B02M13R_c) are about 30 times higher in non-climacteric 'Piel del Sapo' melon than in climacteric 'Védrantais' melon [6]. These characteristics are in accordance with invertase expression differences.

The raffinose oligosaccharides (RFOs) synthesized by photosynthesis can be converted into sucrose and galactose by $\alpha$-galactosidases in fruit tissues. In melon, $\alpha$ galactosidase includes acid and neutral isoenzymes and in young fruit, both can be used to provide energy for the growth metabolism. In mature fruits, sucrose can be stored in the vacuole while galactose can be metabolized to sucrose $[3,6,9]$. In our study, three neutral $\alpha$ galactosidases (CmNAG2, CmNAGLIKE2, CmNAG3) were
DEs (Fig. 7). In the early development stages of 'Yellow' melon, there is an increase of the CmNAG2 expression, that has the highest level in the 20 DAP stage and decreases in 30 and 40 DAP. Though the RNA-seq analyses demonstrated that in the 40 DAP stage the expression of this enzyme was higher than in $10 \mathrm{DAP}$, the $\log 2$ fold change was low (1.07). These differences might be due to individual variations. The CmNAGLIKE2 also has high activity in the beginning of fruit development, while CmNAG3 peaks in the 40 DAP stage. Previous studies showed no DE of neutral $\alpha$-galactosidases in nonclimacteric melon. In climacteric 'Dulce' melon the high activity transcriptional of $C m N A G 2$ was also detected in early stages and low activity of acid $\alpha$-galactosidases in the maturation process [9]. We suggest that the sucrose produced in the catabolic reaction of CmNAG2 and CmNAGLIKE2 in young fruit is recruited in respiration by its conversion to hexoses, evidenced by the high activity of CmAIN2 and CmSUS2 in the same stages. The CmNAG3 has a function in the sucrose accumulation in full-ripe melon.

Another important route of sucrose synthesis is by conversion of galactose-1P into glucose-1P by UDP-glucose epimerase (UGE) $[3,6,9,17]$. In the present study, we observed the increase of $C m U G E 3$ expression in the initial development, followed by subsequent decrease until the full-ripe stage, implying a concomitant gene expression with CmNAG2 (Fig. 7). The activity of both enzymes denotes high sucrose production in young fruits. In climacteric 'Dulce' melon, three UGEs were DE throughout fruit development (CmUGE1, CmUGE2 and CmUGE3), but CmUGE3 expression increased significantly during fruit maturation [9]. The CmUGE3 gene expression or its enzyme activity were not reported in other non-climacteric melon in previous studies $[6,51]$. Antagonistic profile of $C m U G E 3$ is observed in the two melon phenotypes that can be associated with differences in sugar accumulation.

Sucrose-P synthase (SPS) is considered the key gene for sucrose accumulation in fruit ripening $[9,16,18]$. This enzyme catalyzes the reversible transfer of a hexosyl group from UDP-glucose to D-fructose 6-phosphate to form UDP and D-sucrose-6-phosphate [54]. Only CmSPS2 was DE in our study, which has an increase during fruit maturation, reaching the highest levels in colour change melons (30 DAP) (Fig. 7). This is in agreement with gene expression observed in 'Hami' nonclimacteric melon [51]. In climacteric 'Dulce' melon, CmSPS2 is weakly expressed during fruit development, but CmSPS1 rapidly increased from 20 DAP, peaking at late developmental stages [9]. The gene expression of SPS was similar when comparing climacteric and nonclimacteric melon, however different isoenzymes are responsible for syntheses D-sucrose-6-phosphate. The sucrose phosphate phosphatase (SPP) has complementary 
activity with SPS by conversion of sucrose-6-phosphate to sucrose $[3,9,16,18,51]$. In our study only CmSPP1 was DE, having a moderate expression without pronounced differences in the first two stages but with evident decrease in the full-ripe fruit. In non-climacteric 'Hami' melon, the DE of sucrose-P phosphatase was not observed [51]. In climacteric 'Dulce' melon, the CmSPP1 gene was weakly expressed with a slight increase in the 40 DAP stage [9].

\section{Conclusion}

Considering the limited knowledge about molecular mechanisms that act in the ripening process in nonclimacteric melon, studies that involve high-throughput analyses like RNA-seq are paramount to open new perspectives on this matter. Sucrose-cleaving enzymes perform essential mechanisms for the distribution and use of sucrose in fruits. Only sucrose synthase and invertase enzymes can cleave sucrose. CmSUS2 and CmAIN2 are up-regulated in the early development stages of the 'Yellow' melon (Fig. 7), indicating high hexose production, which in turn increases the respiration metabolism and the generation of hexose-based signals. Studies demonstrated that these signals are involved in development processes such as cell division [10]. In addition, the UDP glucose product of sucrose synthase has been implicated in the formation of diverse cell wall polysaccharides [55]. The sucrose substrate for these enzymes is provided by CmNAG2, CmNAGLIKE2 and $C m U G E 3$ transcriptional activity that is high in the same stages. The new putative invertase inhibitor CmINH-LIKE3 (exclusively expressed in 'Yellow' melon) decreases invertase activity in young non-climacteric fruit (Fig. 7), suggesting its importance during nonclimacteric melon fruit development, sucrose accumulation and organic acid content. SPP1 has the highest expression in 20 DAP fruit and CmSPS2 in 30 DAP, both enzymes have a complementary role in sucrose biosynthesis in the intermediate stages (Fig. 7). Finally, CmNAG3 and CmSUS1 have a crucial function in sucrose accumulation in the late stages of fruit development (Fig. 7). Also, the higher expression of CmTPP in the early stages increases the trehalose-6-phosphate conversion to trehalose preventing sucrose synthase inhibition (CmSUS2) (Fig. 7). In contrast, trehalose-6phosphate accumulation by TPS activity inhibits sucrose cleavage in full-ripe melons (Fig. 7).

Many genes within hormone pathways showed differential expression detected by RNA-seq analysis. The hormones also play an essential function in fruit ripening, but the mechanisms are complex and poorly understood. In our study, for IAA, JA, GA, CYT, ABA and BRs signal transduction, most genes are more expressed in young than full-ripe fruit. This characteristic is observed in previous studies [29, 34, 35, 56]. In mature fruit, the auxin response factor, responsive auxin, 9-cisepoxycarotenoid dioxygenase and receptor of ethylene 1 with the highest transcriptional activity were detected and are interesting genes for further melon ripening studies. Furthermore, several studies have demonstrated the integration of sucrose and hormonal pathways including auxin and abscisic acid, as well as epigenetic control (microRNAs and chromosomal modification) acting on fruit development.

This is the first study conducted for non-climacteric 'Yellow' Brazilian commercial melon and the results on sugar metabolism and related pathways during development and ripening contribute to new perspectives in management practices and molecular tools to improve fruit quality.

\section{Methods}

\section{Plant material}

Non-climacteric melon fruit of a 'Yellow' commercial genotype (Cucumis melo, Inodorus group) was cultivated and provided in the different ripening stages by Itaueira Agropecuária SA company in São Paulo (Brazil). Fruit was manually pollinated, and three biological replicates were harvested at different development stages: 10 days after pollination (DAP), 20 DAP, 30 DAP and 40 DAP. For each sampling time, flesh mesocarp was collected, immediately frozen in liquid nitrogen and stored at $-80^{\circ} \mathrm{C}$ until analysis.

\section{Colour, SS (soluble solids) and $\mathrm{pH}$ measurement}

Peel and pulp colour at different ripening stages were measured using a Minolta CR400 colorimeter. The CIE (Commission Internationale de l'Eclairage) L* (lightness), $a^{*}$ (green/red coordinate), b* (blue/yellow coordinate) colour scale was adopted. The angle Hue was calculated by the equation $h^{\circ}=\tan ^{-1}\left(\frac{b *}{a *}\right)$ if $\mathrm{a}^{*}>0$ and $\mathrm{b}^{*}>0$ or by equation $h^{\circ}=180+\tan ^{-1}\left(\frac{b *}{a *}\right)$ if $\mathrm{a}^{*}<0$ or $\mathrm{b}^{*}>0$ [20]. The soluble solids content ( $\left.\mathrm{SS}^{\circ} \mathrm{Brix}\right)$ and the $\mathrm{pH}$ were measured using digital refractometer and automatic $\mathrm{pH}$ matter respectively. Three measurements were made for each fruit and a mean was obtained.

\section{RNA extraction}

Total RNA was extracted in biological triplicate (different fruit) of four development stages using the sodium perchlorate method as described for melon by Campos et al. (2017) [57]. The RNA quality and quantity were determined using Nanovue ${ }^{\mathrm{Tm}}$ spectrophotometer and 1\% agarose gel electrophoresis. Only RNAs that presented A260/A280 ratio $\sim 2.0$, A260/A230 ratio $~ 1.80$ and no discernible degradation were used for RNA-seq and qPCR analyses. 


\section{Preparation of cDNA libraries and RNA-seq}

The cDNA library preparation for RNA-seq analyses was performed to 10 DAP and 40 DAP fruits. Sample RNA quality and concentration for RNA-seq were assessed with the Agilent 2100 Bioanalyzer (Thermo Scientific). Messenger RNA (mRNA) was isolated using the Dynabeads mRNA Direct Micro kit (Life Technologies). Single-end libraries were prepared with the Ion Total RNA-Seq Kit v2, barcoded with the PI ${ }^{\text {mix }}$ Chip Kit v3 at the Federal University of Paraná (Curitiba, BR). After pooling into two-sample groups, the libraries were sequenced (five technical replicas) on an Ion Torrent ${ }^{\mathrm{Tm}}$ (Life Technologies $^{\mathrm{rx}}$ ) using the PI Template $200 \mathrm{bp}$ v3 and Ion PI Sequencing $200 \mathrm{Kit}$ v3. A total of $\sim 27$ million reads were obtained for each sample. The raw sequencing data has been deposited in the NCBI sequence read archive (SRA) under the accession number SRP230494 (https://trace.ncbi.nlm.nih.gov/Traces/ sra/?study=SRP230494) .

\section{Gene expression analysis of RNA-Seq data}

RNA-Seq reads from each biological replicate (2 per 10 DAP and 3 per 40 DAP) were filtered and submitted to adaptor trimming using fastx-toolkits (hannonlab.cshl. edu/fastx_toolkit/) and cutadpt [58] respectively. Reads showing $\geq 80 \%$ of sequenced bases with Phred scores over 20 and more than $50 \mathrm{bp}$ in length were selected. The melon genome (Cucumis melo version v3.6.1) and annotation (gff3 file) provided at https://www.melonomics.net were used as a reference in differential expression analysis. Transcriptome mapping was achieved by using the software Bowtie2 aligner [24]. Gene counts were calculated using featureCounts and only reads with overlapping in a single gene were considered for RNA-seq analysis [59]. Differential expression analyses were carried out applying a statistical test evaluating the negative binomial distribution provided in the $\mathrm{R}$ package DeSeq2 [60]. For each gene, the padj $\leq 0.05$ was considered as the significant threshold. The hierarchical clustering was performed to sample clustering analysis and to evaluate the profile of the top $50 \mathrm{DE}$ genes using the gplots package and heatmap. 2 function available in $\mathrm{R}$.

\section{Gene ontology (GO) and Kyoto encyclopedia of genes and genomes (KEGG)}

Gene ontology term enrichment analysis of DE genes was performed using http://cucurbitgenomics.org/goenrich software (dataset melon DHL92 v3.61), with FDR (false discovery rate) adjusted $p$-value $<0.05$. KEGG pathway enrichment analysis was carried out using DAVID according to the default actions [26]. The pathways of differentially expressed genes were visualized using the 'Pathview' software based on the KO-gene-assignment file and fold change value for each gene under pairwise comparisons
[61]. The degree of $\log 2$ fold changes was highlighted in different colours.

\section{Protein-protein interaction (PPI) network construction and modules mining}

Search Tool for the Retrieval of Interacting Genes/Proteins (STRING) is a database of protein-protein interaction [25]. This database contains direct and physically related interactions between known and predicted proteins and genes. The sources are mainly from (a) experimentally determined (b) text mining in scientific articles and other databases, (c) gene-neighbourhood, (d) gene fusions, (e) co-expression, (f) gene co-occurrence, and (g) protein homology. The system uses a scoring mechanism to give a certain weight to the results and finally gives a comprehensive high throughput analyses [62]. For this analysis we setting the minimum required interaction score 0.400 , none max number of interactors and all interaction sources were selected. The Cytoscape software [63] was used to analyze protein-protein interaction (PPI) generated by STRING. In this study, we set as input the genes 10 DAP-biased fruit separately from genes of 40 DAP-biased fruit. We selected the proteins interactions more relationship with sucrose metabolism from the general network.

\section{Quantitative reverse transcription PCR analysis (RT-qPCR)}

To validate the accuracy of transcriptome profiling, the gene expression of eight transcripts related to the sugar pathway was evaluated by quantitative reverse transcription PCR. Gene specific primers were designed using the 'PrimerBlast' database (https://www.ncbi.nlm.nih.gov/ tools/primer-blast/) (Additional file 3: Table S3). The RPS15 and RPL genes were used with internal control according to Kong et al. (2014) [64]. Also, in these analyses all fruit development stages were considered (10 DAP, 20 DAP, 30 DAP and 40 DAP). Total RNA was treated with the $\mathrm{TURBO}^{\mathrm{iw}}$ DNase kit (Invitrogen) to remove genomic DNA residues from the extraction and it was submitted to cDNA conversion by Maxima $\mathrm{H}$ minus First Strand cDNA Synthesis kit (Thermo Scientific) following the manufacturer's instructions.

RT-qPCR was performed on a LightCycler ${ }^{\circ}$ Nano platform (Roche Diagnostics $\mathrm{GmbH}$, Mannheim, Germany) using $100 \mathrm{ng}$ of cDNA in a reaction containing $1 \mu \mathrm{L}$ of forward and reverse primer $(10 \mu \mathrm{M}), 10 \mu \mathrm{L}$ FastStart Essential DNA Green Master 2X (Roche), in a final volume of $20 \mu \mathrm{L}$. The amplification conditions were performed at $94^{\circ} \mathrm{C}$ for $10 \mathrm{~min}$ and then cycled at $95^{\circ} \mathrm{C}$ for $15 \mathrm{~s}, 55-60^{\circ} \mathrm{C}$ for $20 \mathrm{~s}, 72^{\circ} \mathrm{C}$ for $20 \mathrm{~s}$ for 45 cycles. A melting curve analysis $\left(60^{\circ} \mathrm{C}\right.$ to $\left.99^{\circ} \mathrm{C}\right)$ was performed after the thermal profile to ensure specificity in the amplification. Each assay was performed in triplicates. Relative gene expression analysis was performed using the 
$2^{-\Delta \Delta \mathrm{Ct}}$ method according to Livak and Schmittgen (2001) [65]. Data were converted to a $\log 2$ fold change scale to make the data comparable with the RNA-seq results. Pearson's correlation distance was calculated across 10 DAP and 40 DAP developmental stages.

\section{RT-qPCR statistical analysis}

The $\mathrm{R}$ was used for the statistical analyses. Normality was statistically assessed by the Shapiro-Wilk test [66]. Values that were not normally distributed were transformed by the Box-Cox method [67]. Significant differences among means were determined with the ANOVA $(P \leq 0.05)$ and Tukey's test $(\mathrm{P} \leq 0.05)$. It was not possible to normalize the sucrose synthase 1 (CmSUS1) and sucrose synthase 2 (CmSUS2) genes in RT-qPCR, and the Kruskal-Wallis \& Wilcoxon tests $(\mathrm{P} \leq 0.05)$ were applied in this case.

\section{Supplementary information}

Supplementary information accompanies this paper at https://doi.org/10. 1186/s12864-020-6667-0

\section{Additional file 1: Table S1. Count of number of reads per gene} obtained by featureCounts software. Only reads with overlapping in a sigle gene were considered for RNA-seq analysis; Table S2. RNA-seq data analysis (diferential expression and statistical test).

Additional file 2: Figure S1. PlotMA (Deseq2 R package) shows the $\log 2$ fold changes of young fruits (positive values) and full-ripe fruit (negative values) over the mean of normalized counts for all the samples. Points in red are genes that have significant differential expression (adjusted $p$-value $\leq 0.05$ ). Points that fall out of the window are plotted as open triangles pointing either up or down.

Additional File 3: Table S3. Target genes and reference genes used in RT-qPCR analysis; Figure S2. Pearson's correlation between the 10 DAP and 40 DAP development stage. The expression ratio for RNA-seq and RT-qPCR analysis are represented by log2 fold change.

Additional file 4: Figure S3. Heatmap of the sample-to-sample distances that gives an overview over similarities and dissimilarities between samples $(\mathrm{V}$ is 10 DAP fruit and M is 40 DAP fruit). Dark blue shade indicates higher levels of similarity and light blue indicates higher levels of dissimilarities.

Additional file 5: Table S4. Gene ontology enrichment analysis of young and mature melon DE genes. This analysis was performed using FDR (false discovery rate) adjusted p-value $<0.05$ on DE genes (http:// cucurbitgenomics.org/goenrich).

Additional file 6: Table S5. The top 50 DE genes between young (10 DPA) and mature (40 DAP) fruit samples; Figure S4. Hierarchical clustering analyses of DE top 50 genes between young (10 DPA) and mature (40 DAP) fruit samples. The log2 fold change values were converted by rlog (regularized logarithm) function in Deseq2. Each line represents one gene and the rows are the samples. The colour bar represents the rlog values and ranges from blue (low expression) to red (high expression).

Additional file 7: Table S6. Differential expressed genes present on KEGG enrichment pathways (Fisher exact test $\leq 0.05$ ).

Additional file 8: Figures S5, S6 and Tables S7, S8. Figures represent protein-protein interaction network of young (Figure S4) and mature melon (Figure S5) fruit generated by STRING and Cytoscape analyses. The tables represent the characteristics of the network interaction.

Additional File 9: Tables S9, S10, S11, S12. Characteristics of PPI network interaction of sugar and associated pathways.

Additional File 10: Figures S7, S8, S9. KEGG (Kyoto Encyclopedia of Genes and Genomes) analyses using Pathview software (https://pathview. uncc.edu/) of "starch and sucrose metabolism", "galactose metabolism" and "amino sugar and nucleotide sugar metabolism". The colour bar represents de log2 fold change of the maturation process and ranges from green (up-regulated genes in 40 DAP fruit) to red (up-regulated genes in 10 DAP fruit). The blue letters are enzyme short names described in the KEGG pathway and the purple are enzyme short names that were described in the literature associated with the sugar pathway $[6,9]$. There are protein isoforms that act in the same metabolic route and the information of all $\log 2$ fold change were included.

Additional File 11: Figure S10. Graphics of the normalized gene counts obtained by RNA-seq results (plotCounts function of DESeq2 analysis - differential gene expression analysis based on negative binomial distribution).

Additional File 12: Figure S11. Alignment of protein isoforms related to sugar metabolism or associated pathways. The domains were detected by Pfam database (https://pfam.xfam.org/) and the amino acid sequences were aligned by the MUSCLE algorithm.

\section{Abbreviations}

RNA: Ribonucleic acid; RNA-seq: RNA sequencing; PCR: Polymerase chain reaction; RT-qPCR: Quantitative reverse transcription PCR; DAP: Days after pollination; DNA: Deoxyribonucleic acid; DE: Differentially expressed; RFO: Raffinose family oligosaccharide; Glc: Glucose; NAG: Neutral agalactosidase; AAG: Acid a-galactosidase; GK: Galactokinase; Gal1P: Galactose 1-phosphate; GIc1P: Glucose 1-phosphate; UGGP: UDP-gal/glc pyrophosphorylase; SPS: Sucrose-phosphate synthase; SPP: Sucrosephosphate phosphatase; CIN: Cell wall invertase; HXK: Hexokinase; FK: Fructokinase; SUS: Sucrose synthase; UDP-glc: UDP glucose; NIN: Neutral invertase; AIN: Acid invertase; INH: Invertase inhibitor; NGS: Next generation sequencing; SS: Soluble solids; CIE: Commission Internationale de I'Eclairage; bp: Base pair; DEG: Differentially; expressed genes; GO: Gene ontology; MF: Molecular function; CC: Cellular component; BP: Biological process; ETH: Ethylene; ABA: Abscisic acid; BR: Brassinosteroids; IAA: Auxin; CYT: Cytokinins; GA: Gibberellin; JA: Jasmonic acid; UDP: Uridine diphosphate; T6P: Trehalose-6-phosphate; TPP: Trehalose phosphate phosphatase; miRNA: micro RNA; TPS: Trehalose phosphate synthase; UGE: UDP-glucose epimerase; cDNA: Complementary DNA; mRNA: Messenger RNA; BR: Brazil; NCBI: National Center for Biotechnology Information; KEGG: Kyoto Encyclopedia of Genes and Genomes; PPI: Protein-protein interaction; STRING: Search Tool for the Retrieval of Interacting Genes/Proteins

\section{Acknowledgements}

We are grateful to the Wilson Padilha de Oliveira to help in the laboratory experiments.

\section{Author's contributions}

MOS performed RNA-seq, bioinformatics and statistical analyses. MAS and LR isolated RNA, conduced RT-qPCR and RNA-seg analyses. KKSL, MZTS and GATA performed the library preparation and RNA-seq methodology. MOS, $M A R, L R, C W G, R M E, A R G B$ and RAA interpreted the results and wrote the manuscript. RAA wrote and approved project funding. All of the authors read and approved the final manuscript.

\section{Funding}

This research was supported by Fundação Araucária (Fundação Araucária de Apoio ao Desenvolvimento Científico e Tecnológico, grant number: 23814) and the fellowship was granted by CAPES (Coordenação de Aperfeiçoamento de Pessoal de Nível Superior, grant number: 88887.368562/2019-00).

\section{Availability of data and materials}

The raw sequencing data has been deposited in NCBI sequence read archive (SRA) under the accession number SRP230494 (https://trace.ncbi.nlm.nih.gov/ Traces/sra/?study=SRP230494). All other data analyzed in the present study are included in this article and its additional files.

Ethics approval and consent to participate

Melon plant is widely cultivated and commercially available, and no permits are required for the collection of plant samples. This article did not contain any studies with human, animals and did not involve any endangered or protected species. 


\section{Consent for publication}

Not applicable.

\section{Competing interests}

The authors declare that they have no competing interests.

\section{Author details}

'Laboratório de Biotecnologia Aplicada a Fruticultura, Departamento de Fitotecnia e Fitossanidade, Universidade Estadual de Ponta Grossa, Av. Carlos Cavalcanti, 4748, Ponta Grossa, Paraná 84030-900, Brazil. 'Laboratório de Biologia Molecular Microbiana, Departamento de Biologia Estrutural, Molecular e Genética, Universidade Estadual de Ponta Grossa, Av. Carlos Cavalcanti, 4748, Ponta Grossa, Paraná 84030-900, Brazil. ${ }^{3}$ Departamento de Bioquímica, Centro Politécnico, Universidade Federal do Paraná, Jd. Das Américas, Caixa-Postal 19071, Curitiba, Paraná 81531-990, Brazil.

Received: 19 November 2019 Accepted: 12 March 2020

Published online: 30 March 2020

\section{References}

1. Schaffer AA, Paris HS. Melons, Squashes, and Gourds. Elsevier; 2016.

2. Pitrat M. Genetic Resources of Cucumber. Plant Genet Genomics Crop Model. 2016;20:61-86.

3. Leida C, Moser C, Esteras C, Sulpice R, Lunn JE, de Langen F, et al. Variability of candidate genes, genetic structure and association with sugar accumulation and climacteric behavior in a broad germplasm collection of melon (Cucumis melo L.). BMC Genet. 2015;16:1-17.

4. Pech JC, Purgatto E, Bouzayen M, Latché A. Ethylene and fruit ripening. In: The Plant Hormone Ethylene; 2012.

5. Pech JC, Bouzayen M, Latché A. Climacteric fruit ripening: ethylenedependent and independent regulation of ripening pathways in melon fruit. Plant Sci. 2008;175:114-20.

6. Saladié $M$, Cañizares J, Phillips MA, Rodriguez-Concepcion M, Larrigaudière C, Gibon Y, et al. Comparative transcriptional profiling analysis of developing melon (Cucumis melo L.) fruit from climacteric and nonclimacteric varieties. BMC Genomics. 2015;16:440.

7. Zheng XY, Wolff DW. Ethylene production, shelf-life and evidence of RFLP polymorphisms linked to ethylene genes in melon (Cucumis melo L.). Theor Appl Genet. 2000;101:613-24.

8. Yu K, Xu Q, Da X, Guo F, Ding Y, Deng X. Transcriptome changes during fruit development and ripening of sweet orange (Citrus sinensis). BMC Genomics. 2012;13:10.

9. Dai N, Cohen S, Portnoy V, Tzuri G, Harel-Beja R, Pompan-Lotan M, et al. Metabolism of soluble sugars in developing melon fruit: a global transcriptional view of the metabolic transition to sucrose accumulation. Plant Mol Biol. 2011;76:1-18.

10. Koch K. Sucrose metabolism: regulatory mechanisms and pivotal roles in sugar sensing and plant development. Curr Opin Plant Biol. 2004;7:235-46.

11. Stein O, Granot D. An overview of sucrose synthases in plants. Front Plant Sci. 2019;10:95.

12. Shin AY, Kim YM, Koo N, Lee SM, Nahm S, Kwon SY. Transcriptome analysis of the oriental melon (Cucumis melo L. var. makuwa) during fruit development. PeerJ. 2017;5:e2834

13. Stepansky A, Kovalski I, Schaffer AA, Perl-Treves R. Variation in sugar levels and invertase activity in mature fruit representing a broad spectrum of Cucumis melo genotypes. Genet Resour Crop Evol. 1999;46:53-62.

14. Fernández-Trujillo J, Picó B, García-Mas J, Álvarez JM, Monforte AJ. Breeding for fruit quality in melons. In: Breeding for Fruit Quality; 2011.

15. Ohkawa W, Kanayama Y, Daibo N, Sato T, Nishiyama M, Kanahama K. Metabolic process of the 14C-sugars on the translocation pathways of cucumber plants. Sci Hortic (Amsterdam). 2010;124:46-50.

16. Chayut N, Yuan H, Ohali S, Meir A, Yeselson Y, Portnoy V, et al. A bulk segregant transcriptome analysis reveals metabolic and cellular processes associated with Orange allelic variation and fruit $\beta$-carotene accumulation in melon fruit. BMC Plant Biol. 2015;15:254.

17. Dai N. Cloning and expression analysis of a UDP-Galactose/glucose Pyrophosphorylase from melon fruit provides evidence for the major metabolic pathway of Galactose metabolism in Raffinose oligosaccharide metabolizing plants. Plant Physiol. 2006;142:294-304.

18. Burger $Y$. Schaffer a a. the contribution of sucrose metabolism enzymes to sucrose accumulation in Cucumis melo. J Am Soc Hortic Sci. 2007;132:704-12.
19. Anuário Brasileiro de Horti \& Fruti 2019 - Editora Gazeta. http://www. editoragazeta.com.br/anuario-brasileiro-de-hortifruti-2019/. Accessed 19 Oct 2019.

20. McLellan MR, Lind LR, Kime RW. Hue angle determinations and statistical analysis for multiquadrant Hunter $L$, a, b data. J Food Qual. 1995; 18(3):235-40

21. Kasim R, Kasim MU. Biochemical and color changes of fresh-cut melon (Cucumis melo L. cV. Galia) treated with UV-C. Food Sci Technol. 2014;34(3): 547-51.

22. Garcia-Mas J, Benjak A, Sanseverino W, Bourgeois M, Mir G, Gonzalez VM, et al. The genome of melon (Cucumis melo L.). Proc Natl Acad Sci. 2012;109: $11872-77$.

23. Ruggieri V, Alexiou KG, Morata J, Argyris J, Pujol M, Yano R, et al. An improved assembly and annotation of the melon (Cucumis melo L.) reference genome. Sci Rep. 2018;8:1-9.

24. Langmead B, Salzberg SL. Fast gapped-read alignment with Bowtie 2. Nat Methods. 2012;9(4):357.

25. Szklarczyk D, Franceschini A, Wyder S, Forslund K, Heller D, Huerta-Cepas J, et al. STRING v10: protein-protein interaction networks, integrated over the tree of life. Nucleic Acids Res. 2015:43:D447-52.

26. Huang DW, Sherman BT, Lempicki RA. Systematic and integrative analysis of large gene lists using DAVID bioinformatics resources. Nat Protoc. 2009;4:44-57.

27. Edgar RC. MUSCLE: multiple sequence alignment with high accuracy and high throughput. Nucleic Acids Res. 2004;32:1792-7.

28. White PJ. Recent advances in fruit development and ripening: an overview. J Exp Bot. 2002:53:1995-2000.

29. Wang QH, Zhao C, Zhang M, Li YZ, Shen YY, Guo JX. Transcriptome analysis around the onset of strawberry fruit ripening uncovers an important role of oxidative phosphorylation in ripening. Sci Rep. 2017;7:41477.

30. Pilati S, Perazzolli M, Malossini A, Cestaro A, Demattè $L$, Fontana $P$, et al. Genome-wide transcriptional analysis of grapevine berry ripening reveals a set of genes similarly modulated during three seasons and the occurrence of an oxidative burst at vèraison. BMC Genomics. 2007:8:428.

31. Adams-Phillips L, Barry C, Giovannoni J. Signal transduction systems regulating fruit ripening. Trends Plant Sci. 2004;9:331-8. https://doi.org/10. 1016/j.tplants.2004.05.004.

32. Ayub R, Guis M, Ben AM, Gillot $L$, Roustan JP, Latché $A$, et al. Expression of acc oxidase antisense gene inhibits ripening of cantaloupe melon fruits. Nat Biotechnol. 1996;14:862-6.

33. Fortes AM, Teixeira RT, Agudelo-Romero P. Complex interplay of hormonal signals during grape berry ripening. Molecules. 2015;20:9326-43.

34. Zhang X, Luo G, Wang R, Wang J, Himelrick DG. Growth and developmental responses of seeded and seed less grape berries to shoot girdling. J Am Soc Hortic Sci. 2003;128:316-23.

35. Gansser D, Latza S, Berger RG. Methyl Jasmonates in developing strawberry fruit ( Fragaria ananassa Duch. Cv. Kent). J Agric Food Chem. 1997;45:247780. https://doi.org/10.1021/jf9608940.

36. Leung J, Merlot S, Giraudat J. The arabidopsis ABSCISIC ACID-INSENSITIVE2 $(\mathrm{ABI} 2)$ and $\mathrm{ABI} 1$ genes encode homologous protein phosphatases $2 \mathrm{C}$ involved in abscisic acid signal transduction. Plant Cell. 1997:9:759-71.

37. Giribaldi M, Geny L, Delrot S, Schubert A. Proteomic analysis of the effects of ABA treatments on ripening Vitis vinifera berries. J Exp Bot. 2010;61:244758. https://doi.org/10.1093/jxb/erq079.

38. Koyama K, Sadamatsu K, Goto-Yamamoto N. Abscisic acid stimulated ripening and gene expression in berry skins of the cabernet sauvignon grape. Funct Integr Genomics. 2010;10:367-81.

39. Jia $H$, Wang $Y$, Sun $M, L i B$, Han $Y$, Zhao $Y$, et al. Sucrose functions as a signal involved in the regulation of strawberry fruit development and ripening. New Phytol. 2013;198:453-65.

40. Ayub RA, Reis L, Bosetto L, Lopes PZ, Galvão CW, Etto RM. Brassinosteroid plays a role on pink stage for receptor and transcription factors involved in strawberry fruit ripening. Plant Growth Regul. 2018;84:159-67.

41. Liu Q, Wen CK. Arabidopsis ETR1 and ERS1 differentially repress the ethylene response in combination with other ethylene receptor genes. Plant Physiol. 2012;158:1193-207.

42. Guo S, Sun H, Zhang H, Liu J, Ren Y, Gong G, et al. Comparative transcriptome analysis of cultivated and wild watermelon during fruit development. PLoS One. 2015;10:e0130267.

43. Zhuang JP, Su J, Li XP, Chen WX. Changes in alpha-L-arabinofuranosidase activity in peel and pulp of banana (Musa sp.) fruits during ripening and softening. Zhi wu sheng li yu fen zi sheng wu xue xue bao; J Plant Physiol Mol Biol. 2007;33(2):131-36. 
44. Dai N, Petreikov M, Portnoy V, Katzir N, Pharr DM, Schaffer AA. Cloning and expression analysis of a UDP-Galactose/ glucose Pyrophosphorylase from melon fruit provides evidence for the major metabolic pathway of Galactose metabolism in Raffinose oligosaccharide metabolizing plants. Plant Physiol. 2006;142(1):294-304.

45. Sukumaran RK, Abraham A, Mathew AK. Enzymes for bioenergy. In: Bioresources and Bioprocess in Biotechnology. Springer Singapore; 2017. p. 3-43.

46. Fedosejevs ET, Feil R, Lunn JE, Plaxton WC. The signal metabolite trehalose6-phosphate inhibits the sucrolytic activity of sucrose synthase from developing castor beans. FEBS Lett. 2018;592:2525-32.

47. Figueroa CM, Lunn JE. A tale of two sugars: Trehalose 6-phosphate and sucrose. Plant Physiol. 2016;172:7-27.

48. Jia H, Xie Z, Wang C, Shangguan L, Qian N, Cui M, et al. Abscisic acid, sucrose, and auxin coordinately regulate berry ripening process of the Fujiminori grape. Funct Integr Genomics. 2017:17:441-57.

49. Vashisht D, Mendel G. MicroRNA functions in Plant Embryos doi:https://doi. org/10.1042/BST20130252.

50. Sarnowska E, Gratkowska DM, Sacharowski SP, Cwiek P, Tohge T, Fernie AR, et al. The role of SWI/SNF chromatin remodeling complexes in hormone crosstalk. Trends Plant Sci. 2016;21:594-608.

51. Zhang H, Wang H, Yi H, Zhai W, Wang G, Fu Q. Transcriptome profiling of Cucumis melo fruit development and ripening. Hortic Res. 2016:3:1-10.

52. Wind J, Smeekens S, Hanson J. Sucrose: metabolite and signaling molecule. Phytochemistry. 2010;71:1610-4.

53. Gibson SI. Control of plant development and gene expression by sugar signaling. Curr Opin Plant Biol. 2005;8:93-102.

54. Teck KC, Bujnicki JM, Tan TC, Huynh F, Patel BK, Sivaraman J. The structure of sucrose phosphate synthase from Halothermothrix orenii reveals its mechanism of action and binding mode. Plant Cell. 2008;20:1059-72.

55. Albrecht $\mathrm{G}$, Mustroph A. Localization of sucrose synthase in wheat roots: increased in situ activity of sucrose synthase correlates with cell wall thickening by cellulose deposition under hypoxia. Planta. 2003;217:252-60

56. Luan L-Y, Zhang Z-W, Xi Z-M, Huo S-S, Ma L-N. Brassinosteroids regulate anthocyanin biosynthesis in the ripening of grape berries. South African $S$ Afr J Enol Vitic. 2013;34:196-203.

57. De Campos GS, Ayub RA, Etto RM, Galvão CW, Stroka MA, Inaba J. Highquality total RNA isolation from melon (Cucumis melo L.) fruits rich in polysaccharides. Semin Agrar. 2017;38:2201-8.

58. Martin M. Cutadapt removes adapter sequences from high-throughput sequencing reads. EMBnet.journal. 2011;17:10.

59. Liao Y, Smyth GK, Shi W. featureCounts: an efficient general purpose program for assigning sequence reads to genomic features. Bioinformatics. 2014;30:923-30.

60. Love Ml, Huber W, Anders S. Moderated estimation of fold change and dispersion for RNA-seq data with DESeq2. Genome Biol. 2014;15:550.

61. Luo W, Pant G, Bhavnasi YK, Blanchard SG, Brouwer C. Pathview web: user friendly pathway visualization and data integration. Nucleic Acids Res. 2017;45:W501-8.

62. Woodak SJ, Pu S, Vlasblom J, Séraphin B. Challenges and rewards of interaction proteomics. Mol Cell Proteomics. 2009;8:3-18.

63. Doncheva NT, Morris JH, Gorodkin J, Jensen LJ. Cytoscape StringApp: network analysis and visualization of proteomics data. J Proteome Res. 2019;18:623-32.

64. Kong Q, Gao L, Cao L, Liu Y, Saba H, Huang Y, et al. Assessment of Suitable Reference Genes for Quantitative Gene Expression Studies in Melon Fruits. Front Plant Sci. 2016;7:1178

65. Livak KJ, Schmittgen TD. Analysis of Relative Gene Expression Data Using Real- Time Quantitative PCR and the 2(-Delta Delta C(T)) Method. Methods. 2001;408:402-8.

66. Shapiro SS, Wilk MB. An analysis of variance test for normality (complete samples). Biometrika. 1965;52:591-611.

67. Sakia RM. The box-cox transformation technique: a review. Stat. 1992:41:169

\section{Publisher's Note}

Springer Nature remains neutral with regard to jurisdictional claims in published maps and institutional affiliations.

Ready to submit your research? Choose BMC and benefit from:

- fast, convenient online submission

- thorough peer review by experienced researchers in your field

- rapid publication on acceptance

- support for research data, including large and complex data types

- gold Open Access which fosters wider collaboration and increased citations

- maximum visibility for your research: over $100 \mathrm{M}$ website views per year

At $\mathrm{BMC}$, research is always in progress.

Learn more biomedcentral.com/submissions 